\title{
C-乙烯基鼠李糖苷衍生物的合成及细胞毒活性探究
}

\author{
吉玉 姚辉柳入黄年玉* 刘明国* \\ (三峡大学生物与制药学院 天然产物研究与利用湖北省重点实验室 湖北宜昌 443002)
}

\begin{abstract}
摘要 报道了 $L$-鼠李烯糖与炔丙酯在 $\mathrm{Au}(\mathrm{I})$ 催化下通过分子间串联的 1,3-酰氧基迁移/Ferrier 重排策略, 合成了 $C$-乙烯 基鼠李糖苷衍生物. 通过同位素 $\mathrm{O}^{18}$ 标记实验验证了 $C$-糖基化机理. 产物的绝对构型经 $\mathrm{X}$ 射线单晶衍射分析确定. 利 用噻唑蓝(MTT)法研究了产物的细胞毒活性, 结果显示产物 $3 \mathbf{i}$ 对人体胃癌细胞 HGC-27 具有良好的抑制作用, $\mathrm{IC}_{50}$ 值为 $18.29 \mu \mathrm{mol} \cdot \mathrm{L}^{-1}$. 该方法具有操作简单、高非对映选择性、反应条件温和等优点.
\end{abstract}

关键词 Ferrier 重排; 鼠李糖苷; 细胞毒活性; 非对映选择

\section{Synthesis and Cytotoxic Activity of $C$-Vinyl-rhamnopyranoside Derivatives}

\author{
Ji, Yu Yao, Hui Liu, Yi Huang, Nianyu* Liu, Mingguo* \\ (Key Laboratory of Natural Products Research and Development, College of Biological and Pharmaceutical Sciences, \\ China Three Gorges University, Yichang, Hubei 443002)
}

\begin{abstract}
A novel gold(I)-catalyzed glycosylation method was described to synthesize $C$-vinyl-rhamnopyranoside derivatives using stable propargylic carboxylates and 3,4-di- $O$-acetyl- $L$-rhamnal as starting materials, based on the tandem intermolecular 1,3-acyloxy migration/Ferrier rearrangement. The $C$-glycosylation process has been verified by $\mathrm{O}^{18}$ isotopic labeling experiment, and the absolute configuration of synthesized products was determined by X-ray single crystal diffraction. The cytotoxic activity was investigated by methyl thiazolyl tetrazolium (MTT). It indicates that product $\mathbf{3 i}$ has strong inhibitory effect on human gastric cancer cells HGC-27 with $\mathrm{IC}_{50} 18.29 \mu \mathrm{mol} \cdot \mathrm{L}^{-1}$. The described synthetic method was outstanding with easy-to-operate, high diastereoselectivity, and mild reaction condition.

Keywords Ferrier rearrangement; rhamnopyranoside; cytotoxic activity; diastereoselectivity
\end{abstract}

碳苷作为一类重要的糖苷类化合物, 因其对酸水解 和酶促水解均表现出高稳定性, 被认为是一种稳定的药 效基团, 广泛应用于医药、化工等领域 ${ }^{[1]}$. 其中, 鼠李糖 碳苷衍生物是一类具有显著抗菌活性和抗肿瘤活性的 化合物 ${ }^{[2]}$. 近年来具有生物活性的鼠李糖碳苷类天然产 物和药物分子相继被报道. 例如 Vineomycinone B2 methyl ester ${ }^{[3]} 、$ Polycarcinv $^{[4]} 、 7-O-$-Glycosyl genisteins ${ }^{[5]}$. 因此, 探索鼠李糖碳苷类化合物的高效合成在糖化学和 医药研发领域具有重要的意义.

过渡金属催化偶联反应是构建碳苷键最有效的方 法之一 ${ }^{[6]}$. 除了通过传统的催化偶联反应, 如 Heck、 Suzuki、Stille 以及 Negishi 反应等, 近些年涌现出许多
新型的金属催化体系, 提高了糖苷化反应的立体选择 性 ${ }^{[7]}$. 但是如何简单高效地实现异头碳原子上的区域选 择性和 $\alpha / \beta$ 立体选择性, 依然是当今糖化学研究难点之 一. 其中, 通过过渡金属催化偶联反应合成含 $C$-乙烯基 骨架的糖苷类化合物, 作为一类重要的合成中间体 ${ }^{[8]}$, 以其显著抗菌活性、抗肿瘤活性 ${ }^{[9]}$, 引起合成化学家的 广泛关注. 目前, 合成 $C$-乙烯基鼠李糖苷的例子很少. 例如, 过渡金属催化的烯醇三氟甲磺酸酯与鼠李烯糖的 交叉偶联反应合成 $C$-乙烯基鼠李糖苷 ${ }^{[10]}$, 以及用 Lewis 酸催化的芳基乙炔与鼠李烯糖分子间串联的糖苷化反 应和卤代过程合成 $C$-乙烯基鼠李糖苷 ${ }^{[11]}$. 为了进一步 拓展此类化合物的合成方法, 本工作探究了 $\mathrm{Ph}_{3} \mathrm{PAuCl} /$

\footnotetext{
* Corresponding authors. E-mail: hny115@126.com; mgliu1966@163.com Received March 14, 2020; revised April 19, 2020; published online April 23, 2020. Project supported by the National Natural Science Foundation of China (No. 21602123), and the Youth Talent Development Foundation and Scientific Foundation from Graduate School of China Three Gorges University (No. SDYC2016121).

国家自然科学基金(No. 21602123)及三峡大学青年拔尖人才项目和研究生创新基金(No. SDYC2016121)资助项目.
} 
$\mathrm{AgSbF}_{6}$ 催化下 $L$ - 鼠李烯糖与炔丙酯发生分子间串联的 1,3-酰氧基迁移/Ferrier 重排，探讨了高收率、高立体选 择性地合成 $C$-乙烯基鼠李糖苷的反应.

\section{1 结果与讨论}

为了获得 $C$-乙烯基鼠李糖苷衍生物合成的最优反 应条件, 本工作以 $L$-鼠李烯糖(1a) 和炔丙酯 $(\mathbf{2 a})$ 为模型 反应，首先评估了 $\mathrm{Au}_{2} \mathrm{O}_{3} 、 \mathrm{TiCl}_{4} 、 \mathrm{Ni}(\mathrm{CO})_{4} 、 \mathrm{PdCl}_{2} 、 \mathrm{Ag}_{2} \mathrm{CO}_{3}$ 诱导 $1 \mathbf{a}$ 发生 Ferrier 重排生成 $C$-乙烯基糖苷产物的能力, 然而反应 $24 \mathrm{~h}$ 未检测到目标产物(表 1 , Entries 1 5). 当 使用 $\mathrm{AuCl}_{3}$ 时，室温条件反应 $6 \mathrm{~h}$ 就能以 $21 \%$ 的收率得 到 $C$-乙烯基鼠李糖苷产物 $3 \mathbf{a}$ (表 1 , Entry 6). 这一结果促 使了对金催化剂进一步篮选(表 1, Entries 7～18), 最终 确定 $\mathrm{Ph}_{3} \mathrm{PAuCl}$ 和 $\mathrm{AgSbF}_{6}$ 组合催化剂的催化效果最佳, 能以 $58 \%$ 的收率得到目标产物. 随后对反应溶剂进行笁 选(表 1, Entries 19 24), 发现以 $\mathrm{CH}_{3} \mathrm{NO}_{2}$ 为溶剂时反应 收率高, 达 78\%(表 1, Entry 22). 最终确定了反应的最优 条件: 以 $\mathrm{CH}_{3} \mathrm{NO}_{2}$ 为溶剂, $\mathrm{Ph}_{3} \mathrm{PAuCl} / \mathrm{AgSbF}_{6}(5 \mathrm{~mol} \%)$ 为 催化剂，室温下反应 $6 \mathrm{~h}$.

在最优条件下对炔丙酯的适用范围进行了篮选(表 2 ). 当 $\mathrm{R}^{1}$ 为苯基, $\mathrm{R}^{2}$ 为烷基时, 能够以良好的收率得到 $C$-乙烯基鼠李糖苷衍生物, 且随着烷基 $\left(\mathrm{R}^{2}\right)$ 空间位阻的 增大, 收率有所降低 $(3 \mathbf{a} \sim 3 \mathbf{3})$. 随后考察了苯环上所连 取代基 $\left(\mathrm{R}^{1}\right)$ 的电子效应对反应的影响, 发现供电子效应 的取代基不利于提高衍生物的收率 $(\mathbf{3 g} \sim \mathbf{3 i}, \mathbf{3} \mathbf{j} \sim \mathbf{3 m})$, 而拉电子效应的取代基有利于衍生物收率的提高 $(\mathbf{3 q}$ 3t). 当 $\mathrm{R}^{1}$ 为杂环噻吩时, 能以 $68 \%$ 的收率得到 $3 \mathrm{u}$. 当 $\mathrm{R}^{1} 、 \mathrm{R}^{2}$ 均为烷基时, 也能以较高的收率获得目标产物 $(3 v \sim 3 x)$. 当 $R^{1}$ 为烷基, $R^{2}$ 为芳基时, 同样也能获得较 好的收率 $(\mathbf{3 y})$. 所得产物均为 $\alpha$ 构型, 通过对化合物 $\mathbf{3 g}$ 的 X 射线单晶衍射分析也进一步验证了这一结果(图 1).

为了研究鼠李烯糖保护基空间位阻的改变对反应 的影响, 设计了实验(表 3). 以苯甲酰基保护的鼠李烯糖 (1b 1f) 在上述最优条件下反应，能以 $73 \% \sim 78 \%$ 的收 率、高立体选择性得到 $C$-乙烯基鼠李糖苷衍生 4. 烯糖 保护基空间位阻的增大对反应收率和选择性几乎没有 影响. 通过上述实验说明, 该反应具有广泛的官能团耐 受性和高非对映选择性，为简单高效制备 $C$-乙烯基鼠 李糖苷衍生物提拱了新方法.

随后将该方法扩展到岩藻烯糖(1g)时, 发现对岩藻 烯糖同样适用, 以高非对映选择性得到预期的 $C$-乙烯 基岩藻糖苷衍生物 5 (Eq. 1), 其收率高于由鼠李烯糖反 应时的产物. 这可能是由于岩藻烯糖 C4 上基团的空间 取向，在糖苷化过程中更有利于联烯中间体的亲核进攻.

通过查阅相关文献 ${ }^{[12]}$, 提出了如下可能反应机理
表 1 模型反应条件优化 ${ }^{a}$

Table 1 Optimization of reaction conditions for the model reaction

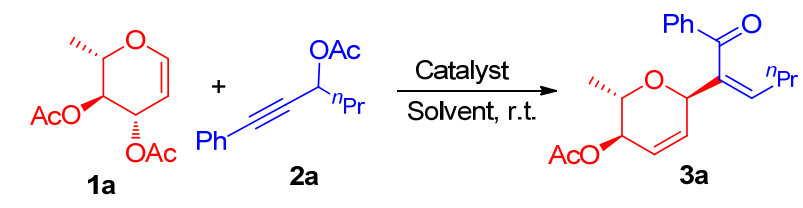

\begin{tabular}{|c|c|c|c|c|}
\hline Entry & Catalyst & Solvent & Time/h & Yield $^{b} / \%$ \\
\hline 1 & $\mathrm{Au}_{2} \mathrm{O}_{3}$ & $\mathrm{CH}_{2} \mathrm{Cl}_{2}$ & 24 & N.D. \\
\hline 2 & $\mathrm{TiCl}_{4}$ & $\mathrm{CH}_{2} \mathrm{Cl}_{2}$ & 24 & N.D. \\
\hline 3 & $\mathrm{Ni}(\mathrm{CO})_{4}$ & $\mathrm{CH}_{2} \mathrm{Cl}_{2}$ & 24 & N.D. \\
\hline 4 & $\mathrm{PdCl}_{2}$ & $\mathrm{CH}_{2} \mathrm{Cl}_{2}$ & 24 & N.D. \\
\hline 5 & $\mathrm{Ag}_{2} \mathrm{CO}_{3}$ & $\mathrm{CH}_{2} \mathrm{Cl}_{2}$ & 24 & N.D. \\
\hline 6 & $\mathrm{AuCl}_{3}$ & $\mathrm{CH}_{2} \mathrm{Cl}_{2}$ & 6 & 21 \\
\hline 7 & $\mathrm{AuBr}_{3}$ & $\mathrm{CH}_{2} \mathrm{Cl}_{2}$ & 6 & 12 \\
\hline 8 & $\mathrm{AuCl}$ & $\mathrm{CH}_{2} \mathrm{Cl}_{2}$ & 6 & 31 \\
\hline 9 & $\mathrm{Ph}_{3} \mathrm{PAuCl}$ & $\mathrm{CH}_{2} \mathrm{Cl}_{2}$ & 24 & N.D. \\
\hline 10 & $\mathrm{AgSbF}_{6}$ & $\mathrm{CH}_{2} \mathrm{Cl}_{2}$ & 24 & N.D. \\
\hline 11 & $\mathrm{Au}(\mathrm{CO}) \mathrm{Cl} / \mathrm{AgSbF}_{6}$ & $\mathrm{CH}_{2} \mathrm{Cl}_{2}$ & 6 & 24 \\
\hline 12 & $\mathrm{Ph}_{3} \mathrm{PAuCl} / \mathrm{AgSbF}_{6}$ & $\mathrm{CH}_{2} \mathrm{Cl}_{2}$ & 6 & 58 \\
\hline 13 & $\mathrm{Ph}_{3} \mathrm{PAuNTf}_{2} / \mathrm{AgSbF}_{6}$ & $\mathrm{CH}_{2} \mathrm{Cl}_{2}$ & 6 & 42 \\
\hline 14 & $\mathrm{Ph}_{3} \mathrm{PAuCl} / \mathrm{AgNO}_{3}$ & $\mathrm{CH}_{2} \mathrm{Cl}_{2}$ & 6 & 28 \\
\hline 15 & $\mathrm{Ph}_{3} \mathrm{PAuCl} / \mathrm{CH}_{3} \mathrm{CO}_{2} \mathrm{Ag}$ & $\mathrm{CH}_{2} \mathrm{Cl}_{2}$ & 6 & 37 \\
\hline 16 & $\mathrm{Ph}_{3} \mathrm{PAuCl} / \mathrm{Ag}_{2} \mathrm{CO}_{3}$ & $\mathrm{CH}_{2} \mathrm{Cl}_{2}$ & 6 & 45 \\
\hline 17 & $\mathrm{Ph}_{3} \mathrm{PAuCl} / \mathrm{Ag}_{3} \mathrm{PO}_{4}$ & $\mathrm{CH}_{2} \mathrm{Cl}_{2}$ & 6 & 48 \\
\hline 18 & $\left(\mathrm{C}_{5} \mathrm{H}_{5} \mathrm{~N}\right) \mathrm{AuCl}_{3} / \mathrm{AgSbF}_{6}$ & $\mathrm{CH}_{2} \mathrm{Cl}_{2}$ & 6 & 46 \\
\hline 19 & $\mathrm{Ph}_{3} \mathrm{PAuCl} / \mathrm{AgSbF}_{6}$ & DMF & 6 & N.D. \\
\hline 20 & $\mathrm{Ph}_{3} \mathrm{PAuCl} / \mathrm{AgSbF}_{6}$ & $\mathrm{CHCl}_{3}$ & 6 & 36 \\
\hline 21 & $\mathrm{Ph}_{3} \mathrm{PAuCl} / \mathrm{AgSbF}_{6}$ & Toluene & 6 & 25 \\
\hline 22 & $\mathrm{Ph}_{3} \mathrm{PAuCl} / \mathrm{AgSbF}_{6}$ & $\mathrm{CH}_{3} \mathrm{NO}_{2}$ & 6 & 78 \\
\hline 23 & $\mathrm{Ph}_{3} \mathrm{PAuCl} / \mathrm{AgSbF}_{6}$ & $\mathrm{CH}_{3} \mathrm{CN}$ & 6 & 12 \\
\hline 24 & $\mathrm{Ph}_{3} \mathrm{PAuCl} / \mathrm{AgSbF}_{6}$ & EtOAc & 6 & N.D. \\
\hline
\end{tabular}

${ }^{a}$ General conditions: catalyst $(5 \mathrm{~mol} \%)$ was added into the mixture of $1 \mathbf{a}(107$ $\mathrm{mg}, 0.5 \mathrm{mmol})$ and $\mathbf{2 a}(113 \mathrm{mg}, 0.5 \mathrm{mmol})$ in corresponding solvent $(5 \mathrm{~mL})$.

${ }^{b}$ Isolated yield. N.D. $=$ not detected.

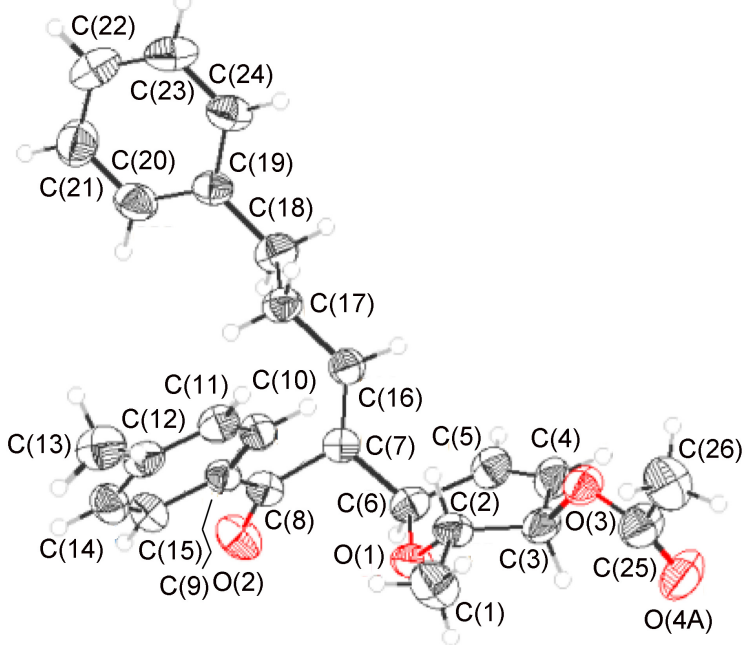

图 1 化合物 $\mathbf{3 g}$ 的晶体结构

Figure 1 X-ray single crystal structure of $\mathbf{3 g}$ 
表 $2 C$-乙烯基鼠李糖苷衍生物的底物拓展

Table 2 Substrate scope for $C$-vinyl-rhamnopyranoside derivatives

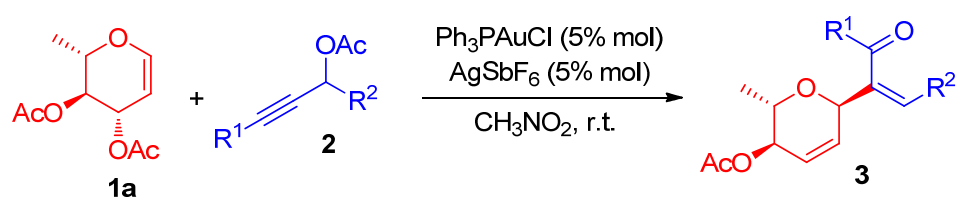

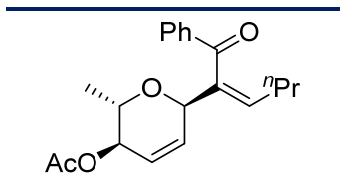

$3 a, 78 \%$<smiles>CC(=O)OC1C=CC(/C(=C/c2ccccc2)C(=O)c2ccccc2)O[C@@H]1C</smiles>

3f, $74 \%$<smiles>CC/C=C(/C(=O)c1ccc(CC)cc1)C1C=CC(OC(C)=O)[C@@H](C)O1</smiles>

3k, $69 \%$<smiles>C[C@H]1O[C@H](C(=CC(C)(C)C)C(=O)c2ccc(C(C)(C)C)cc2)C=C[C@@H]1OC(C)(C)C</smiles>

$3 p, 70 \%$<smiles>CC(=O)O[C@H]1C=C[C@@H](C(=CC(C)C)C(=O)c2ccsc2)O[C@@H]1C</smiles>

$3 u, 68 \%$

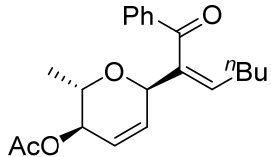

3b, $78 \%$<smiles>CC(=O)O[C@H]1C=C[C@@H](C(=Cc2ccccc2)C(=O)c2ccc(C)cc2)O[C@@H]1C</smiles>

3g, $65 \%$

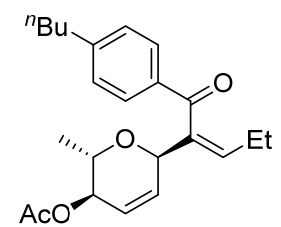

3I, $73 \%$

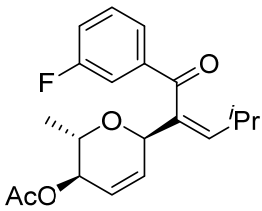

$3 q, 86 \%$

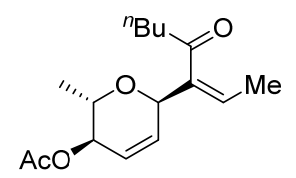

$3 v, 81 \%$

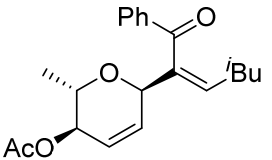

3c, $76 \%$

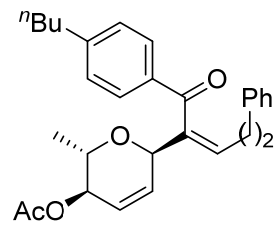

$3 \mathrm{~h}, 66 \%$<smiles>CC/C=C(\C(=O)c1ccc(OC)cc1)[C@@H]1C=C[C@@H](OC(C)=O)[C@H](C)O1</smiles>

$3 \mathrm{~m}, 64 \%$<smiles>CCCCC(=O)C(=CC(C)C)[C@@H]1C=C[C@H](OC(C)=O)[C@@H](C)O1</smiles>

$3 r, 84 \%$

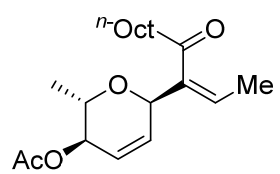

$3 w, 74 \%$

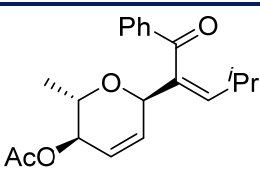

3d, $72 \%$<smiles>COc1ccc(C(=O)C(=Cc2ccccc2)[C@H]2C=C[C@H](OC(C)=O)[C@@H](C)O2)cc1</smiles>

$3 i, 52 \%$

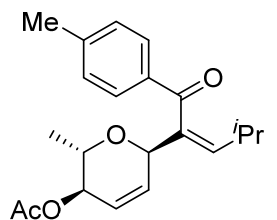

$3 n, 63 \%$

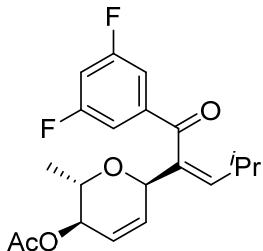

3s, $92 \%$

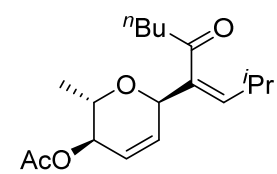

$3 x, 76 \%$<smiles>CC(=O)O[C@H]1C=C[C@H](/C(=C/CBr)C(=O)c2ccccc2)O[C@@H]1C</smiles>

3e, $65 \%$<smiles>CC/C=C(\C(=O)c1ccc(C)cc1)[C@H]1C=C[C@@H](OC(C)C)[C@@H](C)O1</smiles>

3j, $68 \%$<smiles>CCc1ccc(C(=O)C(=CC(C)(C)CC)[C@H]2C=C[C@H](OC(C)=O)[C@@H](C)O2)cc1</smiles>

3o, $72 \%$<smiles>CC(=O)O[C@H]1C=C[C@@H](C(=CC(C)(C)C)C(=O)c2ccc(F)cc2)O[C@@H]1C</smiles>

$3 t, 82 \%$
(Scheme 1). 在反应过程中, 金催化剂不仅可以作为路 易斯酸, 促使烯糖发生 Ferrier 重排生成氧鎓离子 $\mathbf{C}$, 也 能活化炔键发生分子内环化生成 $\mathbf{A}$, 随后发生 1,3-酰氧 基迁移生成联烯 $\mathbf{B}$, 进而对 $\mathbf{C}$ 发生亲核进攻, 氧鎓离子 解离得到 $C$-乙烯基鼠李糖苷 $\mathbf{E}$.

为了验证反应机理经历炔丙酯迁移过程, 设计了同 位素标记实验. 利用 $L$ - 鼠李烯糖 $1 \mathrm{a}$ 与 ${ }^{18} \mathrm{O}$ 标记的炔丙酯 $\mathbf{2} \mathbf{a}-{ }^{18} \mathbf{O}^{[13]}$ 在最优条件下进行反应, 从 $\mathbf{3 a}-{ }^{18} \mathbf{O}$ 的 ${ }^{13} \mathrm{C}$ NMR 谱中可以清楚地看到 ${ }^{18} \mathrm{O}$ 标记的羰基碳在 $\delta 198.76$ 处有 信号峰. 同时, HRMS 也证实了 $\mathbf{3 a}-{ }^{18} \mathbf{O}$ 的存在. 这一结 果充分证明炔丙酯在反应过程中发生了 1,3-酰氧基迁移 (Eq. 2).

通常含有鼠李糖碳苷骨架的化合物具有良好的抗
肿瘤活性，促使我们对合成的新化合物 $3 \sim 5$ 进行细胞 毒活性研究. 利用噻唑蓝(MTT)法, 选择三种人体癌细 胞株 [胃癌细胞(HGC-27)、宫颈癌细胞(Caski)和肝癌细 胞(Hep-G2)]和人胃粘膜细胞(GES-1)进行体外细胞毒活 性测试 ${ }^{[14]}$. 通过 SPSS 13.0 软件分别计算化合物对于不 同细胞的 $\mathrm{IC}_{50}$ 值. 测试结果(表 4)表明, 所有化合物对人 胃粘膜细胞均表现出低毒性. 化合物 $\mathbf{3 i}$ 具有显著的体外 胃癌细胞抑制增长活性, 其 $\mathrm{IC}_{50}$ 值为 $18.29 \mu \mathrm{mol} \cdot \mathrm{L}^{-1}$. 随着 3i 浓度的增加, 细胞毒活性也随之增加(图 2).

\section{2 结论}

在 $\mathrm{Au}(\mathrm{I})$ 催化作用下, 炔丙酯与鼠李烯糖发生分子 间串联的 1,3-酰氧基迁移/Ferrier 重排反应，以 52\% 
表 3 C-乙烯基鼠李糖苷衍生物的底物扩展

Table 3 Substrate expansion of $C$-vinyl-rhamnopyranoside derivatives<smiles>[R]C=C(C([R])=O)[C@H]1C=C[C@@H](OC([R])=O)[C@@H](C)O1</smiles>

\begin{tabular}{clcc}
\hline Entry & \multicolumn{1}{c}{$\mathrm{R}^{1}(\mathbf{1})$} & $\mathrm{R}^{2}$ & Yield/\% (4) \\
\hline 1 & $\mathrm{Ph}(\mathbf{1 b})$ & $\mathrm{Et}$ & $76(\mathbf{4 b a})$ \\
2 & $\mathrm{Ph}(\mathbf{1 b})$ & ${ }^{i} \mathrm{Bu}$ & $73(\mathbf{4 b b})$ \\
3 & $2-\mathrm{ClC}_{6} \mathrm{H}_{4}(\mathbf{1 c})$ & ${ }^{i} \mathrm{Bu}$ & $74(\mathbf{4 c a})$ \\
4 & $3-\mathrm{ClC}_{6} \mathrm{H}_{4}(\mathbf{1 d})$ & ${ }^{i} \mathrm{Bu}$ & $75(\mathbf{4 d a})$ \\
5 & $2-\mathrm{FC}_{6} \mathrm{H}_{4}(\mathbf{1 e})$ & ${ }^{i} \mathrm{Bu}$ & $76(\mathbf{4 e a})$ \\
6 & $4-\mathrm{FC}_{6} \mathrm{H}_{4}(\mathbf{1 f})$ & ${ }^{i} \mathrm{Bu}$ & $78(\mathbf{4 f a})$ \\
\hline
\end{tabular}<smiles>[R]C#CC([R])OC(C)=O</smiles>

19<smiles>CC/C=C(/C(=O)c1ccccc1)[C@@H]1C=C[C@@H](OC(C)=O)[C@H](C)O1</smiles>

5a, $96 \%$<smiles></smiles>

5 b, $92 \%$

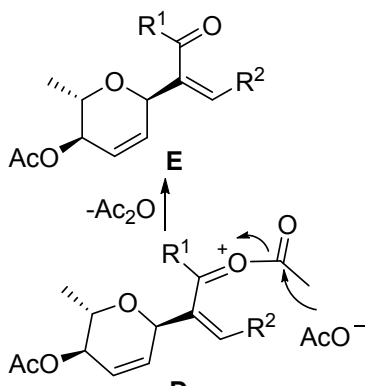

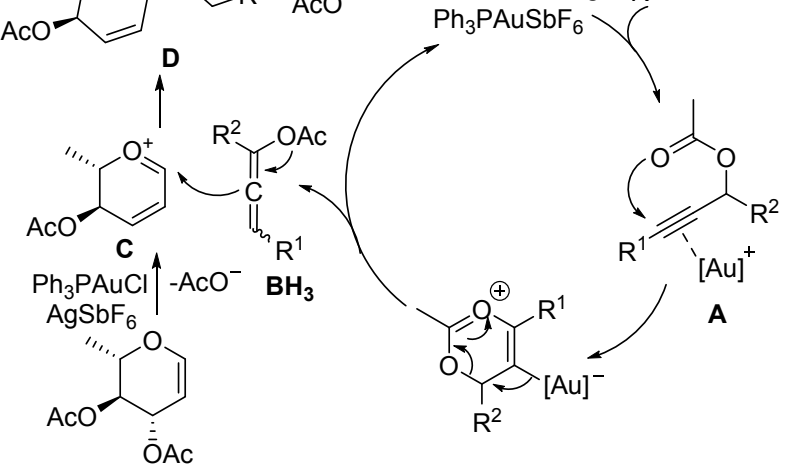

图式 1 可能的反应机理

Scheme 1 Possible reaction mechanism

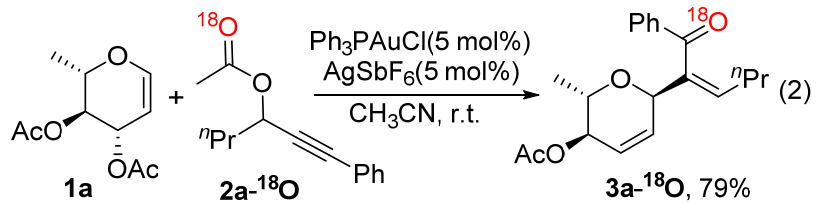

表 4 目标化合物的细胞毒活性

Table 4 Cytotoxic activity of target compounds

\begin{tabular}{lcccc}
\hline \multirow{2}{*}{ 化合物 } & \multicolumn{4}{c}{$\mathrm{IC}_{50}$ 值 $/\left(\mu \mathrm{mol} \cdot \mathrm{L}^{-1}\right)$} \\
\cline { 2 - 5 } & 胃癌细胞 & 宫颈癌细胞 & 肝癌细胞 & 人胃粘膜细胞 \\
\hline $\mathbf{3 a \sim 3 h}$ & $>100$ & $>100$ & $>100$ & $>100$ \\
$\mathbf{3 i}$ & 18.29 & $>100$ & $>100$ & $>100$ \\
$\mathbf{3 j} \sim \mathbf{3 o}$ & $>100$ & $>100$ & $>100$ & $>100$ \\
$\mathbf{3 p}$ & 82.91 & $>100$ & $>100$ & $>100$ \\
$\mathbf{3 q} \sim \mathbf{3 y}$ & $>100$ & $>100$ & $>100$ & $>100$ \\
$\mathbf{4 b a}$ & $>100$ & $>100$ & $>100$ & $>100$ \\
$\mathbf{4 b b}$ & 90.21 & $>100$ & $>100$ & $>100$ \\
$\mathbf{4} \mathbf{c a} \sim \mathbf{4 f a}$ & $>100$ & $>100$ & $>100$ & $>100$ \\
$\mathbf{5 a} \sim \mathbf{5 b}$ & $>100$ & $>100$ & $>100$ & $>100$ \\
\hline
\end{tabular}

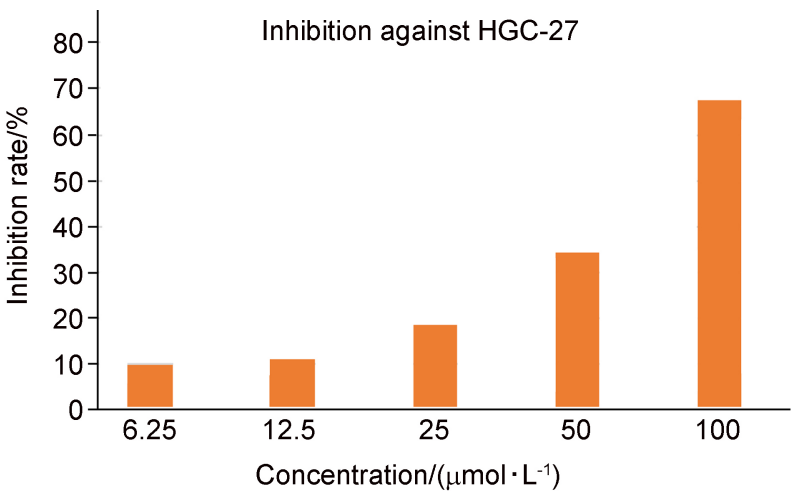

图 2 化合物 $\mathbf{3 i}$ 的胃癌细胞毒活性

Figure 2 Cytotoxities against HGC-27 cells of compound 3i

92\%的收率、高非对映选择性地合成了一系列 $C$-乙烯基 鼠李糖苷衍生物. 通过 ${ }^{1} \mathrm{H} N M R,{ }^{13} \mathrm{C} N M R$ 以及 $X$ 射线 单晶衍射分析确定所得产物的绝对构型. 将该方法扩展 到其它烯糖底物来探索其更多的潜在药用价值将在未 来的研究中相继开展.

\section{3 实验部分}

\section{1 仪器与试剂}

所有反应均在氮气氛围中进行. 反应溶剂均参照标 准方法进行无水无氧处理. Bruker AVANCE III $400 \mathrm{MHz}$ 核磁共振仪, Oxford diffraction Gemini E 型单晶衍射仪, Agilent 1290-6540 UHPLC Q-Tof 型质谱仪. 化合物 $\mathbf{1 a} \sim \mathbf{1} \mathbf{f}^{[15]}$ 和 $\mathbf{2} \mathbf{a} \sim \mathbf{2} \mathbf{y}^{[16]}$ 参考文献方法制备. 其他商品化 试剂购买后, 无需纯化直接使用.

\section{2 实验方法}

\section{2 .1 化合物 $\mathbf{3 a} \sim \mathbf{3 y}$ 的合成}

在 $25 \mathrm{~mL}$ 的 Schlenk 管内, 依次加入 $L$-鼠李烯糖 $\mathbf{1 a}$ 
(107 mg, $0.5 \mathrm{mmol}$ ), 炔丙酯 2 (0.5 mmol), $\mathrm{CH}_{3} \mathrm{NO}_{2}$ (5 $\mathrm{mL})$, 室温搅拌 $10 \mathrm{~min}$ 后, 迅速加入 $\mathrm{Ph}_{3} \mathrm{PAuCl}(12.3$ $\mathrm{mg}, 5 \mathrm{~mol} \%$ ) 和 $\mathrm{AgSbF}_{6}$ (6.5 mg, $5 \mathrm{~mol} \%$ ). 室温下搅拌 6 $\mathrm{h}$, 薄层色谱(TLC)检测反应完全. 用砂芯漏斗过滤, 减 压浓缩除去溶剂. 通过硅胶快速色谱法(石油醚/乙酸乙 酯, $V: V=20: 1)$ 纯化残余物, 得到 $C$-乙烯基鼠李糖苷 衍生物 3.

( $2 S, 3 R, 6 R)$-2-甲基-6-((Z)-1-苯基-2-己烯-1-酮)-3,6二氢- $2 \mathrm{H}$-吡喃-3-基乙酸酯(3a): 无色油状, $128 \mathrm{mg}$, 产率 $78 \% .{ }^{1} \mathrm{H}$ NMR $\left(\mathrm{CDCl}_{3}, 400 \mathrm{MHz}\right) \delta: 7.93 \sim 7.90(\mathrm{~m}, 2 \mathrm{H})$, $7.61 \sim 7.56(\mathrm{~m}, 1 \mathrm{H}), 7.50 \sim 7.46(\mathrm{~m}, 2 \mathrm{H}), 5.98$ (ddd, $J=$ $10.3,3.0,1.6 \mathrm{~Hz}, 1 \mathrm{H}), 5.91 \sim 5.87(\mathrm{~m}, 2 \mathrm{H}), 5.08(\mathrm{br}, 1 \mathrm{H})$, $5.00(\mathrm{dq}, J=7.6,2.2 \mathrm{~Hz}, 1 \mathrm{H}), 3.85 \sim 3.79(\mathrm{~m}, 1 \mathrm{H}), 2.09$ (s, $3 \mathrm{H}), 1.93 \sim 1.86(\mathrm{~m}, 2 \mathrm{H}), 1.42 \sim 1.33(\mathrm{~m}, 2 \mathrm{H}), 1.10(\mathrm{~d}, J=$ $6.4 \mathrm{~Hz}, 3 \mathrm{H}), 0.81(\mathrm{t}, J=7.4 \mathrm{~Hz}, 3 \mathrm{H}) ;{ }^{13} \mathrm{C} \mathrm{NMR}\left(\mathrm{CDCl}_{3}\right.$, $100 \mathrm{MHz}) \delta: 198.9,170.6,138.5,137.5,135.3,133.4$, $130.0,129.3,128.7,126.6,73.1,70.4,66.9,31.7,22.4$, $21.2,17.5,13.7$. HRMS calcd for $\mathrm{C}_{20} \mathrm{H}_{24} \mathrm{O}_{4} \mathrm{Na}[\mathrm{M}+\mathrm{Na}]^{+}$ 351.1567 , found 351.1565 .

( $2 S, 3 R, 6 R)$-2-甲基-6-((Z)-1-苯基-2-庚基-1-酮)-3,6二氢- $2 \mathrm{H}$-吡喃-3-基乙酸酯(3b): 无色油状, $133 \mathrm{mg}$, 产 率 78\%. ${ }^{1} \mathrm{H}$ NMR $\left(\mathrm{CDCl}_{3}, 400 \mathrm{MHz}\right) \delta: 7.93 \sim 7.90(\mathrm{~m}$, 2H), $7.60 \sim 7.56(\mathrm{~m}, 1 \mathrm{H}), 7.49 \sim 7.45(\mathrm{~m}, 2 \mathrm{H}), 5.98$ (ddd, $J=10.3,3.0,1.6 \mathrm{~Hz}, 1 \mathrm{H}), 5.91 \sim 5.87(\mathrm{~m}, 2 \mathrm{H}), 5.08(\mathrm{br}$, $1 \mathrm{H}), 5.00(\mathrm{dq}, J=7.6,2.2 \mathrm{~Hz}, 1 \mathrm{H}), 3.85 \sim 3.79(\mathrm{~m}, 1 \mathrm{H})$, $2.09(\mathrm{~s}, 3 \mathrm{H}), 1.97 \sim 1.86(\mathrm{~m}, 2 \mathrm{H}), 1.34 \sim 1.29(\mathrm{~m}, 2 \mathrm{H})$, $1.22 \sim 1.16(\mathrm{~m}, 2 \mathrm{H}), 1.11$ (d, $J=6.3 \mathrm{~Hz}, 3 \mathrm{H}), 0.78$ (t, $J=$ $7.3 \mathrm{~Hz}, 3 \mathrm{H}) ;{ }^{13} \mathrm{C} \mathrm{NMR}\left(\mathrm{CDCl}_{3}, 100 \mathrm{MHz}\right) \delta: 198.9,170.6$, $138.4,137.5,135.5,133.4,130.1,129.2,128.6,126.6$, $73.1,70.4,66.9,31.3,29.5,22.2,21.2,17.5,13.8$. HRMS calcd for $\mathrm{C}_{21} \mathrm{H}_{26} \mathrm{O}_{4} \mathrm{Na}[\mathrm{M}+\mathrm{Na}]^{+}$365.1723, found 365.1724 .

( $2 S, 3 R, 6 R$ )-2-甲基-6-((Z)-5-甲基-1-苯基-2-己烯-1酮)-3,6-二氢- $2 H$-吡喃-3-基乙酸酯(3c): 无色油状, 130 $\mathrm{mg}$, 产率 76\%. ${ }^{1} \mathrm{H} \mathrm{NMR}\left(\mathrm{CDCl}_{3}, 400 \mathrm{MHz}\right) \delta: 7.75 \sim 7.73$ (m, 2H), $7.56 \sim 7.52(\mathrm{~m}, 1 \mathrm{H}), 7.46 \sim 7.42(\mathrm{~m}, 2 \mathrm{H}), 6.42(\mathrm{t}$, $J=7.6 \mathrm{~Hz}, 1 \mathrm{H}), 6.06(\mathrm{dd}, J=10.2,1.9 \mathrm{~Hz}, 1 \mathrm{H}), 5.89$ (ddd, $J=10.3,4.8,2.6 \mathrm{~Hz}, 1 \mathrm{H}), 5.40 \sim 5.38(\mathrm{~m}, 1 \mathrm{H}), 4.88 \sim 4.86$ $(\mathrm{m}, 1 \mathrm{H}), 4.16(\mathrm{qd}, J=6.8,2.4 \mathrm{~Hz}, 1 \mathrm{H}), 2.47(\mathrm{t}, J=7.2$ $\mathrm{Hz}, 2 \mathrm{H}), 2.09$ (s, 3H), $1.74 \sim 1.67(\mathrm{~m}, 1 \mathrm{H}), 1.32(\mathrm{~d}, J=6.8$ $\mathrm{Hz}, 3 \mathrm{H}), 0.95$ (d, $J=6.6 \mathrm{~Hz}, 3 \mathrm{H}), 0.91$ (d, $J=6.6 \mathrm{~Hz}, 3 \mathrm{H})$;

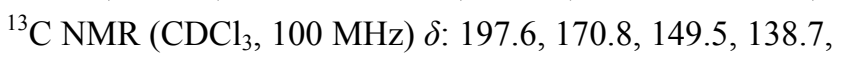
138.1, 133.7, 132.0, 129.6, 128.1, 120.6, 71.0, 68.4, 66.0, 37.3, 28.7, 22.7, 22.6, 21.2, 16.0. HRMS calcd for $\mathrm{C}_{21} \mathrm{H}_{26} \mathrm{O}_{4} \mathrm{Na}[\mathrm{M}+\mathrm{Na}]^{+}$365.1723, found 365.1724.
(2S,3R,6R)-2-甲基-6-((Z)-4-甲基-1-苯基-2-戊烯-1酮)-3,6-二氢- $2 H$-吡喃-3-基乙酸酯(3d): 无色油状, 118 $\mathrm{mg}$, 产率 72\%. ${ }^{1} \mathrm{H} \mathrm{NMR}\left(\mathrm{CDCl}_{3}, 400 \mathrm{MHz}\right) \delta: 7.94 \sim 7.91$ $(\mathrm{m}, 2 \mathrm{H}), 7.60 \sim 7.56(\mathrm{~m}, 1 \mathrm{H}), 7.50 \sim 7.46(\mathrm{~m}, 2 \mathrm{H}), 5.97$ (ddd, $J=10.3,2.9,1.6 \mathrm{~Hz}, 1 \mathrm{H}), 5.90 \sim 5.87(\mathrm{~m}, 1 \mathrm{H}), 5.67$ (dd, $J=10.6,1.2 \mathrm{~Hz}, 1 \mathrm{H}), 5.05 \sim 5.03(\mathrm{~m}, 1 \mathrm{H}), 4.99(\mathrm{dq}$, $J=7.6,2.2 \mathrm{~Hz}, 1 \mathrm{H}), 3.83 \sim 3.77(\mathrm{~m}, 1 \mathrm{H}), 2.28 \sim 2.19(\mathrm{~m}$, $1 \mathrm{H}), 2.08$ (s, 3H), 1.11 (d, $J=6.4 \mathrm{~Hz}, 3 \mathrm{H}), 0.94$ (d, $J=6.6$ $\mathrm{Hz}, 3 \mathrm{H}), 0.90$ (d, $J=6.6 \mathrm{~Hz}, 3 \mathrm{H}) ;{ }^{13} \mathrm{C} \mathrm{NMR}\left(\mathrm{CDCl}_{3}, 100\right.$ MHz) $\delta: 198.7,170.5,141.5,137.5,136.2,133.4,130.0$, 129.2, 128.6, 126.6, 73.1, 70.4, 66.9, 29.0, 22.7, 22.4, 21.2, 17.5. HRMS calcd for $\mathrm{C}_{20} \mathrm{H}_{24} \mathrm{O}_{4} \mathrm{Na}[\mathrm{M}+\mathrm{Na}]^{+} 351.1567$, found 351.1566 .

( $2 S, 3 R, 6 R$ )-2-甲基-6-((Z)-4,4-二甲基-1-苯基-2-戊 烯-1-酮)-3,6-二氢-2 $H$-吡喃-3-基乙酸酯(3e): 无色油状, $111 \mathrm{mg}$, 产率 65\%. ${ }^{1} \mathrm{H} \mathrm{NMR}\left(\mathrm{CDCl}_{3}, 400 \mathrm{MHz}\right) \delta: 7.98 \sim$ $7.96(\mathrm{~m}, 2 \mathrm{H}), 7.59 \sim 7.56(\mathrm{~m}, 1 \mathrm{H}), 7.49 \sim 7.46(\mathrm{~m}, 2 \mathrm{H})$, 5.96 (ddd, $J=10.3,2.9,1.6 \mathrm{~Hz}, 1 \mathrm{H}), 5.86(\mathrm{dt}, J=10.4,2.1$ $\mathrm{Hz}, 1 \mathrm{H}), 5.73(\mathrm{~d}, J=1.1 \mathrm{~Hz}, 1 \mathrm{H}), 4.94(\mathrm{dq}, J=7.3,2.2 \mathrm{~Hz}$, 1H), 4.85 (br, 1H), 3.78 3.71 (m, 1H), 2.05 (s, 3H), 1.08 $(\mathrm{d}, J=6.4 \mathrm{~Hz}, 3 \mathrm{H}), 1.01(\mathrm{~s}, 9 \mathrm{H}) ;{ }^{13} \mathrm{C}$ NMR $\left(\mathrm{CDCl}_{3}, 100\right.$ MHz) $\delta: 199.4,170.6,143.3,137.5,135.1,133.3,130.2$, 129.6, 128.5, 126.3, 74.4, 70.3, 67.0, 34.1, 30.2, 21.1, 17.4. HRMS calcd for $\mathrm{C}_{21} \mathrm{H}_{26} \mathrm{O}_{4} \mathrm{Na}[\mathrm{M}+\mathrm{Na}]^{+} 365.1723$, found 365.1736 .

( $2 S, 3 R, 6 R)$-2- 甲基 -6-((Z)-1,5-二苯基-2-戊烯 -1酮)-3,6-二氢- $2 H$-吡喃-3-基乙酸酯(3f): 黄色油状, 144 $\mathrm{mg}$, 产率 74\%. ${ }^{1} \mathrm{H}$ NMR $\left(\mathrm{CDCl}_{3}, 400 \mathrm{MHz}\right) \delta: 7.79(\mathrm{~d}$, $J=7.5 \mathrm{~Hz}, 2 \mathrm{H}), 7.58 \sim 7.54(\mathrm{~m}, 1 \mathrm{H}), 7.44 \sim 7.41(\mathrm{~m}, 2 \mathrm{H})$, $7.26 \sim 7.15(\mathrm{~m}, 3 \mathrm{H}), 7.01(\mathrm{~d}, J=7.2 \mathrm{~Hz}, 2 \mathrm{H}), 5.96 \sim 5.87$ (m, 3H), 5.04 (br, 1H), 5.00 (dd, $J=7.6,1.2 \mathrm{~Hz}, 1 \mathrm{H})$, $3.82 \sim 3.75(\mathrm{~m}, 1 \mathrm{H}), 2.66(\mathrm{t}, J=7.6 \mathrm{~Hz}, 2 \mathrm{H}), 2.27 \sim 2.21$ (m, 2H), 2.10 (s, 3H), 1.09 (d, $J=6.3 \mathrm{~Hz}, 3 \mathrm{H}) ;{ }^{13} \mathrm{C} \mathrm{NMR}$ $\left(\mathrm{CDCl}_{3}, 100 \mathrm{MHz}\right) \delta: 198.7,170.5,140.8,139.1,137.3$, $134.2,133.5,129.9,129.2,128.7,128.5,128.4,126.8$, 126.2, 126.1, 73.1, 70.4, 66.8, 35.4, 35.2, 31.6, 21.2, 17.6. HRMS calcd for $\mathrm{C}_{21} \mathrm{H}_{27} \mathrm{O}_{4}[\mathrm{M}+\mathrm{H}]^{+}$391.1904, found 391.1910.

(2S,3R,6R)-2-甲基-6-(( $Z$ )-1-(对甲苯基)-5-苯基-2-戊 烯-1-酮)-3,6-二氢-2 $H$-吡喃-3-基乙酸酯(3g): 无色油状, $131 \mathrm{mg}$, 产率 65\%. ${ }^{1} \mathrm{H}$ NMR $\left(\mathrm{CDCl}_{3}, 400 \mathrm{MHz}\right) \delta: 7.72 \sim$ $7.70(\mathrm{~m}, 2 \mathrm{H}), 7.24 \sim 7.15(\mathrm{~m}, 5 \mathrm{H}), 7.03 \sim 7.00(\mathrm{~m}, 2 \mathrm{H})$, 5.94 (ddd, $J=10.3,2.8,1.5 \mathrm{~Hz}, 1 \mathrm{H}), 5.91 \sim 5.85(\mathrm{~m}, 2 \mathrm{H})$, 5.03 (br, $1 \mathrm{H}), 4.99(\mathrm{dq}, J=7.6,2.0 \mathrm{~Hz}, 1 \mathrm{H}), 3.80 \sim 3.76$ (m, $1 \mathrm{H}), 2.65(\mathrm{t}, J=7.6 \mathrm{~Hz}, 2 \mathrm{H}), 2.41(\mathrm{~s}, 3 \mathrm{H}), 2.27 \sim$ 
2.21(m, 2H), $2.10(\mathrm{~s}, 3 \mathrm{H}), 1.09(\mathrm{~d}, J=6.3 \mathrm{~Hz}, 3 \mathrm{H}) ;{ }^{13} \mathrm{C}$ NMR $\left(\mathrm{CDCl}_{3}, 100 \mathrm{MHz}\right) \delta: 196.9,170.5,147.9,147.5$, $140.9,138.2,134.9,133.3,129.6,128.1,128.1,127.9$, $125.8,120.3,70.7,68.0,65.9,35.3,34.9,33.0,30.0,22.0$, 20.6, 20.5, 15.6, 13.6. HRMS calcd for $\mathrm{C}_{26} \mathrm{H}_{28} \mathrm{O}_{4} \mathrm{Na}[\mathrm{M}+$ $\mathrm{Na}]^{+}$427.1880, found 427.1870.

( $2 S, 3 R, 6 R)$-2-甲基-6-( $(Z)$-1-(4-丁基苯基)-5-苯基-2戊烯-1-酮)-3,6-二氢- $2 H$-吡喃-3-基乙酸酯(3h): 无色油 状, $147 \mathrm{mg}$, 产率 $66 \% .{ }^{1} \mathrm{H} \mathrm{NMR}\left(\mathrm{CDCl}_{3}, 400 \mathrm{MHz}\right) \delta$ : $7.66 \sim 7.63(\mathrm{~m}, 2 \mathrm{H}), 7.30 \sim 7.26(\mathrm{~m}, 3 \mathrm{H}), 7.22 \sim 7.18(\mathrm{~m}$, $4 \mathrm{H}), 6.41(\mathrm{t}, J=7.5 \mathrm{~Hz}, 1 \mathrm{H}), 5.98(\mathrm{dd}, J=10.2,1.4 \mathrm{~Hz}$, $1 \mathrm{H}), 5.89$ (ddd, $J=10.2,4.1,2.1 \mathrm{~Hz}, 1 \mathrm{H}), 5.35 \sim 5.34(\mathrm{~m}$, $1 \mathrm{H}), 4.84 \sim 4.82(\mathrm{~m}, 1 \mathrm{H}), 4.14(\mathrm{qd}, J=6.6,2.3 \mathrm{~Hz}, 1 \mathrm{H})$, $2.98 \sim 2.83(\mathrm{~m}, 2 \mathrm{H}), 2.80 \sim 2.69(\mathrm{~m}, 2 \mathrm{H}), 2.66(\mathrm{t}, J=7.7$ $\mathrm{Hz}, 2 \mathrm{H}), 1.83(\mathrm{~s}, 3 \mathrm{H}), 1.66 \sim 1.60(\mathrm{~m}, 2 \mathrm{H}), 1.40 \sim 1.34(\mathrm{~m}$, 2H), 1.30 (d, $J=6.8 \mathrm{~Hz}, 3 \mathrm{H}), 0.94$ (t, $J=7.3 \mathrm{~Hz}, 3 \mathrm{H}) ;{ }^{13} \mathrm{C}$ NMR $\left(\mathrm{CDCl}_{3}, 100 \mathrm{MHz}\right) \delta: 197.2,170.9,148.2,147.8$, $141.2,138.5,135.2,133.6,129.9,128.5,128.4,128.2$, 126.1, 120.6, 71.0, 68.4, 66.3, 35.7, 35.3, 33.3, 30.2, 22.4, 20.9, 15.9, 13.9. HRMS calcd for $\mathrm{C}_{29} \mathrm{H}_{34} \mathrm{O}_{4} \mathrm{Na}[\mathrm{M}+\mathrm{Na}]^{+}$ 469.2349, found 469.2351.

( $2 S, 3 R, 6 R)$-2-甲基-6-(( $Z$ )-1-(4-甲氧基苯基)-5-苯基2-戊烯-1-酮)-3,6-二氢- $2 H$-吡喃-3-基乙酸酯(3i): 无色油 状, $109 \mathrm{mg}$, 产率 $52 \% .{ }^{1} \mathrm{H} \mathrm{NMR}\left(\mathrm{CDCl}_{3}, 400 \mathrm{MHz}\right) \delta$ : $7.72 \sim 7.68(\mathrm{~m}, 2 \mathrm{H}), 7.31 \sim 7.28(\mathrm{~m}, 2 \mathrm{H}), 7.22 \sim 7.18(\mathrm{~m}$, $3 \mathrm{H}), 6.91 \sim 6.87(\mathrm{~m}, 2 \mathrm{H}), 6.32(\mathrm{t}, J=7.4 \mathrm{~Hz}, 1 \mathrm{H}), 5.98$ (dd, $J=10.3,1.3 \mathrm{~Hz}, 1 \mathrm{H}), 5.87$ (ddd, $J=10.2,4.6,2.4 \mathrm{~Hz}$, $1 \mathrm{H}), 5.30 \sim 5.29(\mathrm{~m}, 1 \mathrm{H}), 4.85 \sim 4.82(\mathrm{~m}, 1 \mathrm{H}), 4.11(\mathrm{qd}$, $J=6.7,2.5 \mathrm{~Hz}, 1 \mathrm{H}), 3.87(\mathrm{~s}, 3 \mathrm{H}), 2.96 \sim 2.82(\mathrm{~m}, 2 \mathrm{H})$, $2.81 \sim 2.71(\mathrm{~m}, 2 \mathrm{H}) 1.85(\mathrm{~s}, 3 \mathrm{H}), 1.28(\mathrm{~d}, J=6.8 \mathrm{~Hz}, 3 \mathrm{H})$; ${ }^{13} \mathrm{C} \mathrm{NMR}\left(\mathrm{CDCl}_{3}, 100 \mathrm{MHz}\right) \delta: 196.3,170.9,163.1,146.7$, $141.2,138.5,133.6,132.2,130.2,128.5,128.5,126.1$, $120.8,113.4,70.9,68.4,66.6,55.5,35.3,30.1,20.9,16.0$. HRMS calcd for $\mathrm{C}_{26} \mathrm{H}_{28} \mathrm{O}_{5} \mathrm{Na}[\mathrm{M}+\mathrm{Na}]^{+} 443.1829$, found 443.1844 .

(2S,3R,6R)-2-甲基-6-(( $Z$ )-1-(4-甲基苯基)-2-戊烯-1酮)-3,6-二氢- $2 H$-吡喃-3-基乙酸酯(3j): 无色油状, 112 $\mathrm{mg}$, 产率 68\%. ${ }^{1} \mathrm{H} \mathrm{NMR}\left(\mathrm{CDCl}_{3}, 400 \mathrm{MHz}\right) \delta: 7.83 \sim 7.82$ $(\mathrm{m}, 2 \mathrm{H}), 7.28 \sim 7.26(\mathrm{~m}, 2 \mathrm{H}), 5.98(\mathrm{ddd}, J=10.3,3.0,1.6$ $\mathrm{Hz}, 1 \mathrm{H}), 5.90 \sim 5.82(\mathrm{~m}, 2 \mathrm{H}), 5.07 \sim 5.06(\mathrm{~m}, 1 \mathrm{H}), 4.12$ $(\mathrm{qd}, J=4.1,2.1 \mathrm{~Hz}, 1 \mathrm{H}), 3.85 \sim 3.79(\mathrm{~m}, 1 \mathrm{H}), 2.43(\mathrm{~s}, 3 \mathrm{H})$, 2.09 (s, 3H), 1.96 1.88 (m, 2H), $1.11(\mathrm{~d}, J=6.4 \mathrm{~Hz}, 3 \mathrm{H})$, $0.93(\mathrm{t}, J=7.5 \mathrm{~Hz}, 3 \mathrm{H}) ;{ }^{13} \mathrm{C} \mathrm{NMR}\left(\mathrm{CDCl}_{3}, 100 \mathrm{MHz}\right) \delta$ : $197.5,171.0,150.8,149.2,137.8,135.6,133.8,130.2$, $127.8,120.9,70.9,68.7,66.6,29.1,22.0,21.3,16.2,15.4$,
13.6. HRMS calcd for $\mathrm{C}_{20} \mathrm{H}_{24^{-}}-\mathrm{O}_{4} \mathrm{Na}[\mathrm{M}+\mathrm{Na}]^{+} 351.1567$, found 351.1569 .

( $2 S, 3 R, 6 R)$-2-甲基-6-(( $Z$ )-1-(4-乙基苯基)-2-戊烯-1酮)-3,6-二氢- $2 H$-吡喃-3-基乙酸酯(3k): 无色油状， 118 $\mathrm{mg}$, 产率 69\%. ${ }^{1} \mathrm{H} \mathrm{NMR}\left(\mathrm{CDCl}_{3}, 400 \mathrm{MHz}\right) \delta: 7.71 \sim 7.68$ $(\mathrm{m}, 2 \mathrm{H}), 7.27(\mathrm{~s}, 1 \mathrm{H}), 7.25(\mathrm{~s}, 1 \mathrm{H}), 6.35(\mathrm{t}, J=7.7 \mathrm{~Hz}, 1 \mathrm{H})$, $6.06(\mathrm{dd}, J=10.2,1.2 \mathrm{~Hz}, 1 \mathrm{H}), 5.88$ (ddd, $J=10.2,4.6,2.4$ $\mathrm{Hz}, 1 \mathrm{H}), 5.38 \sim 5.36(\mathrm{~m}, 1 \mathrm{H}), 4.88 \sim 4.85(\mathrm{~m}, 1 \mathrm{H}), 4.13$ $(\mathrm{qd}, J=6.8,2.8 \mathrm{~Hz}, 1 \mathrm{H}), 2.71(\mathrm{q}, J=7.6 \mathrm{~Hz}, 2 \mathrm{H}), 2.61 \sim$ $2.53(\mathrm{~s}, 3 \mathrm{H}), 1.30(\mathrm{~d}, J=6.8 \mathrm{~Hz}, 3 \mathrm{H}), 1.25$ (d, $J=2.6 \mathrm{~Hz}$, $3 \mathrm{H}), 1.04$ (t, $J=7.5 \mathrm{~Hz}, 3 \mathrm{H}) ;{ }^{13} \mathrm{C} \mathrm{NMR}\left(\mathrm{CDCl}_{3}, 100 \mathrm{MHz}\right)$ $\delta: 197.5,171.0,150.8,149.2,137.8,135.6,133.8,130.2$, $127.8,120.9,70.9,68.7,66.6,29.1,22.0,21.3,16.2,15.4$, 13.6. HRMS calcd for $\mathrm{C}_{21} \mathrm{H}_{26} \mathrm{O}_{4} \mathrm{Na}[\mathrm{M}+\mathrm{Na}]^{+}$365.1723, found 365.1725 .

(2S,3R,6R)-2-甲基-6-(( $Z$ )-1-(4-丁基苯基)-2-戊烯-1酮)-3,6-二氢- $2 H$-吡喃-3-基乙酸酯(3I): 无色油状， 135 $\mathrm{mg}$, 产率 73\%. ${ }^{1} \mathrm{H} \mathrm{NMR}\left(\mathrm{CDCl}_{3}, 400 \mathrm{MHz}\right) \delta: 7.69 \sim 7.67$ $(\mathrm{m}, 2 \mathrm{H}), 7.27 \sim 7.23(\mathrm{~m}, 2 \mathrm{H}), 6.36(\mathrm{t}, J=7.6 \mathrm{~Hz}, 1 \mathrm{H}), 6.06$ (dd, $J=10.2,1.3 \mathrm{~Hz}, 1 \mathrm{H}), 5.89$ (ddd, $J=10.2,4.5,2.5 \mathrm{~Hz}$, $1 \mathrm{H}), 5.38 \sim 5.36(\mathrm{~m}, 1 \mathrm{H}), 4.88 \sim 4.86(\mathrm{~m}, 1 \mathrm{H}), 4.14(\mathrm{qd}$, $J=6.7,3.0 \mathrm{~Hz}, 1 \mathrm{H}), 2.67(\mathrm{t}, J=7.6 \mathrm{~Hz}, 2 \mathrm{H}), 2.61 \sim 2.54$ $(\mathrm{m}, 2 \mathrm{H}), 2.08(\mathrm{~s}, 3 \mathrm{H}), 1.64 \sim 1.60(\mathrm{~m}, 2 \mathrm{H}), 1.42 \sim 1.34(\mathrm{~m}$, 2H), 1.30 (d, $J=6.8 \mathrm{~Hz}, 3 \mathrm{H}), 1.05$ (t, $J=7.5 \mathrm{~Hz}, 3 \mathrm{H}), 0.94$ $(\mathrm{t}, J=7.4 \mathrm{~Hz}, 3 \mathrm{H}) ;{ }^{13} \mathrm{C} \mathrm{NMR}\left(\mathrm{CDCl}_{3}, 100 \mathrm{MHz}\right) \delta: 197.4$, $170.8,150.7,150.7,147.8,137.7,135.4,133.7,130.0$, $128.2,120.7,70.8,68.6,66.5,35.7,33.3,22.4,21.9,21.2$, 16.1, 14.0, 13.5. HRMS calcd for $\mathrm{C}_{23} \mathrm{H}_{30} \mathrm{O}_{4} \mathrm{Na}[\mathrm{M}+\mathrm{Na}]^{+}$ 393.2036, found 393.2038.

( $2 S, 3 R, 6 R)$-2-甲基-6-((Z)-1-(4-甲氧基苯基)-2-戊烯1-酮)-3,6-二氢- $2 H$-吡喃-3-基乙酸酯 $(\mathbf{3 m})$ : 无色油状, $110 \mathrm{mg}$, 产率 $64 \% .{ }^{1} \mathrm{H} \mathrm{NMR}\left(\mathrm{CDCl}_{3}, 400 \mathrm{MHz}\right) \delta: 7.80 \sim$ $7.77(\mathrm{~m}, 2 \mathrm{H}), 6.94 \sim 6.90(\mathrm{~m}, 2 \mathrm{H}), 6.26(\mathrm{t}, J=7.6 \mathrm{~Hz}, 1 \mathrm{H})$, $6.06(\mathrm{dd}, J=10.2,1.2 \mathrm{~Hz}, 1 \mathrm{H}), 5.87$ (ddd, $J=10.3,4.1,2.3$ $\mathrm{Hz}, 1 \mathrm{H}), 5.32 \sim 5.31(\mathrm{~m}, 1 \mathrm{H}), 4.87 \sim 4.85(\mathrm{~m}, 1 \mathrm{H}), 4.10$ (qd, $J=6.5,3.1 \mathrm{~Hz}, 1 \mathrm{H}), 3.86(\mathrm{~s}, 3 \mathrm{H}), 2.58 \sim 2.51(\mathrm{~m}, 2 \mathrm{H})$, $2.06(\mathrm{~s}, 3 \mathrm{H}), 1.28(\mathrm{~d}, J=6.8 \mathrm{~Hz}, 3 \mathrm{H}), 1.04(\mathrm{t}, J=7.5 \mathrm{~Hz}$, $3 \mathrm{H}) ;{ }^{13} \mathrm{C} \mathrm{NMR}\left(\mathrm{CDCl}_{3}, 100 \mathrm{MHz}\right) \delta: 196.5,170.8,163.0$, $148.9,137.7,133.5,132.2,130.4,121.0,113.4,70.6,68.7$, 66.8, 55.5, 21.8, 21.2, 16.2, 13.6. HRMS calcd for $\mathrm{C}_{20} \mathrm{H}_{24} \mathrm{O}_{5} \mathrm{Na}[\mathrm{M}+\mathrm{Na}]^{+}$367.1516, found 367.1531.

( $2 S, 3 R, 6 R)$-2-甲基-6-(( $Z$ )-1-(4-甲基苯基)-2-戊烯-1酮)-3,6-二氢-2 $H$-吡喃-3-基乙酸酯(3n): 黄色油状, 108 $\mathrm{mg}$, 产率 63\%. ${ }^{1} \mathrm{H} \mathrm{NMR}\left(\mathrm{CDCl}_{3}, 400 \mathrm{MHz}\right) \delta: 7.68 \sim 7.65$ $(\mathrm{m}, 2 \mathrm{H}), 7.25 \sim 7.23(\mathrm{~m}, 2 \mathrm{H}), 6.12 \sim 6.10(\mathrm{~m}, 1 \mathrm{H}), 6.07$ 
(dd, $J=10.2,1.3 \mathrm{~Hz}, 1 \mathrm{H}$ ), 5.88 (ddd, $J=10.2,4.3,2.5 \mathrm{~Hz}$, $1 \mathrm{H}), 5.36 \sim 5.34(\mathrm{~m}, 1 \mathrm{H}), 4.88 \sim 4.85(\mathrm{~m}, 1 \mathrm{H}), 4.12(\mathrm{qd}$, $J=6.8,3.0 \mathrm{~Hz}, 1 \mathrm{H}), 3.42 \sim 3.33(\mathrm{~m}, 1 \mathrm{H}), 2.42(\mathrm{~s}, 3 \mathrm{H})$, 2.07 (s, 3H), 1.29 (d, $J=6.8 \mathrm{~Hz}, 3 \mathrm{H}), 1.07$ (d, $J=6.5 \mathrm{~Hz}$, $3 \mathrm{H}), 0.98(\mathrm{~d}, J=6.6 \mathrm{~Hz}, 3 \mathrm{H}) ;{ }^{13} \mathrm{C} \mathrm{NMR}\left(\mathrm{CDCl}_{3}, 100 \mathrm{MHz}\right)$ $\delta: 197.5,170.8,155.3,142.9,135.8,135.2,133.8,130.1$, $128.9,120.7,70.6,68.6,66.7,27.5,22.5,22.2,21.6,21.2$, 16.1. HRMS calcd for $\mathrm{C}_{21} \mathrm{H}_{26} \mathrm{O}_{4} \mathrm{Na}[\mathrm{M}+\mathrm{Na}]^{+} 365.1723$, found 365.1725 .

( $2 S, 3 R, 6 R$ )-2-甲基-6-((Z)-1-(4-乙基苯基)-5-甲基-2己烯-1-酮)-3,6-二氢- $2 H$-吡喃-3-基乙酸酯(30): 黄色油 状, $133 \mathrm{mg}$, 产率 72\%. ${ }^{1} \mathrm{H}$ NMR $\left(\mathrm{CDCl}_{3}, 400 \mathrm{MHz}\right) \delta$ : $7.84(\mathrm{~d}, J=8.1 \mathrm{~Hz}, 2 \mathrm{H}), 7.29$ (d, $J=8.1 \mathrm{~Hz}, 2 \mathrm{H}), 5.98$ (ddd, $J=10.3,3.2,1.8 \mathrm{~Hz}, 1 \mathrm{H}), 5.91 \sim 5.86(\mathrm{~m}, 2 \mathrm{H}), 5.07$ (br, $1 \mathrm{H}), 5.00$ (dd, $J=7.7,1.8 \mathrm{~Hz}, 1 \mathrm{H}), 3.86 \sim 3.79(\mathrm{~m}$, $1 \mathrm{H}), 2.72(\mathrm{q}, J=7.6 \mathrm{~Hz}, 2 \mathrm{H}), 2.09(\mathrm{~s}, 3 \mathrm{H}), 1.91 \sim 1.77(\mathrm{~m}$, $2 \mathrm{H}), 1.68 \sim 1.61(\mathrm{~m}, 1 \mathrm{H}), 1.27(\mathrm{t}, J=7.6 \mathrm{~Hz}, 3 \mathrm{H}), 1.11(\mathrm{~d}$, $J=6.2 \mathrm{~Hz}, 3 \mathrm{H}), 0.82(\mathrm{t}, J=6.7 \mathrm{~Hz}, 6 \mathrm{H}) ;{ }^{13} \mathrm{C} \mathrm{NMR}\left(\mathrm{CDCl}_{3}\right.$, $100 \mathrm{MHz}) \delta: 198.5,170.6,150.4,139.2,135.0,133.7$, $130.0,129.5128 .1,126.5,73.2,70.4,66.7,38.5,29.0$, 28.5, 22.4, 22.1, 17.5, 15.1. HRMS calcd for $\mathrm{C}_{23} \mathrm{H}_{30} \mathrm{O}_{4} \mathrm{Na}$ $[\mathrm{M}+\mathrm{Na}]^{+}$393.2036, found 393.2034.

(2S,3R,6R)-2-甲基-6-((Z)-1-(4-丁基苯基)-5-甲基-2己烯-1-酮)-3,6-二氢- $2 H$-吡喃-3-基乙酸酯(3p): 无色油 状, $139 \mathrm{mg}$, 产率 $70 \% .{ }^{1} \mathrm{H}$ NMR $\left(\mathrm{CDCl}_{3}, 400 \mathrm{MHz}\right) \delta$ : $7.70 \sim 7.68(\mathrm{~m}, 2 \mathrm{H}), 7.25 \sim 7.23(\mathrm{~m}, 2 \mathrm{H}), 6.39(\mathrm{t}, J=7.6$ $\mathrm{Hz}, 1 \mathrm{H}$ ), 6.06 (dd, $J=10.3,1.2 \mathrm{~Hz}, 1 \mathrm{H}), 5.88$ (ddd, $J=$ $10.2,4.2,2.5 \mathrm{~Hz}, 1 \mathrm{H}), 5.37 \sim 5.35(\mathrm{~m}, 1 \mathrm{H}), 4.88 \sim 4.86(\mathrm{~m}$, $1 \mathrm{H}), 4.14(\mathrm{qd}, J=6.7,2.7 \mathrm{~Hz}, 1 \mathrm{H}), 2.66(\mathrm{t}, J=7.7 \mathrm{~Hz}$, $2 \mathrm{H}), 2.46(\mathrm{t}, J=7.2 \mathrm{~Hz}, 2 \mathrm{H}), 2.08(\mathrm{~s}, 3 \mathrm{H}), 1.76 \sim 1.68(\mathrm{~m}$, $1 \mathrm{H}), 1.64 \sim 1.59(\mathrm{~m}, 2 \mathrm{H}), 1.42 \sim 1.34(\mathrm{~m}, 2 \mathrm{H}), 1.31(\mathrm{~d}, J=$ $6.8 \mathrm{~Hz}, 3 \mathrm{H}), 0.96 \sim 0.91(\mathrm{~m}, 9 \mathrm{H}) ;{ }^{13} \mathrm{C} \mathrm{NMR}\left(\mathrm{CDCl}_{3}, 100\right.$ MHz) $\delta: 197.4,170.8,148.6,147.8,138.6,135.4,133.8$, $129.9,128.2,120.5,70.9,68.5,66.2,37.2,35.6,33.3,28.6$, 22.7, 22.6, 22.3, 21.2, 16.0, 13.9. HRMS calcd for $\mathrm{C}_{23} \mathrm{H}_{30} \mathrm{O}_{4} \mathrm{Na}[\mathrm{M}+\mathrm{Na}]^{+} 421.2349$, found 421.2351 .

( $2 S, 3 R, 6 R$ )-2-甲基-6-( $(Z)$-4-甲基-1-(3-氟苯基)-2-戊 烯-1-酮)-3,6-二氢-2 $H$-吡喃-3-基乙酸酯(3q): 黄色油状, $149 \mathrm{mg}$, 产率 86\%. ${ }^{1} \mathrm{H}$ NMR $\left(\mathrm{CDCl}_{3}, 400 \mathrm{MHz}\right) \delta: 7.71$ (dt, $J=7.7,1.2 \mathrm{~Hz}, 1 \mathrm{H}), 7.61$ (ddd, $J=9.3,2.6,1.5 \mathrm{~Hz}$, $1 \mathrm{H}), 7.49 \sim 7.44(\mathrm{~m}, 1 \mathrm{H}), 7.31 \sim 7.26(\mathrm{~m}, 1 \mathrm{H}), 5.97$ (ddd, $J=10.3,2.8,1.5 \mathrm{~Hz}, 1 \mathrm{H}), 5.90$ (dt, $J=10.3,2.0 \mathrm{~Hz}, 1 \mathrm{H})$, $5.68(\mathrm{dd}, J=10.7,1.2 \mathrm{~Hz}, 1 \mathrm{H}), 5.03 \sim 5.01(\mathrm{~m}, 1 \mathrm{H}), 4.99$ (dq, $J=7.3,3.7,2.2 \mathrm{~Hz}, 1 \mathrm{H}), 3.82 \sim 3.75(\mathrm{~m}, 1 \mathrm{H}), 2.27 \sim$ $2.15(\mathrm{~m}, 1 \mathrm{H}), 2.08(\mathrm{~m}, 3 \mathrm{H}), 1.12(\mathrm{~d}, J=6.4 \mathrm{~Hz}, 3 \mathrm{H}), 0.95$ (d, $J=6.5 \mathrm{~Hz}, 3 \mathrm{H}), 0.91(\mathrm{~d}, J=6.6 \mathrm{~Hz}, 3 \mathrm{H}) ;{ }^{13} \mathrm{C} \mathrm{NMR}$ $\left(\mathrm{CDCl}_{3}, 100 \mathrm{MHz}\right) \delta: 197.4,170.5,162.8\left(\mathrm{~d}, J_{\mathrm{C}-\mathrm{F}}=247.0\right.$ $\mathrm{Hz}), 142.1,139.7\left(\mathrm{~d}, J_{\mathrm{C}-\mathrm{F}}=6.0 \mathrm{~Hz}\right), 135.9,130.19(\mathrm{~d}$, $\left.J_{\mathrm{C}-\mathrm{F}}=8.0 \mathrm{~Hz}\right), 129.8,126.6,125.0,120.5,120.6,120.3$ $115.7\left(\mathrm{~d}, J_{\mathrm{C}-\mathrm{F}}=22.0 \mathrm{~Hz}\right), 72.9,70.2,67.0,29.1,22.6,22.4$, 21.1, 17.4. HRMS calcd for $\mathrm{C}_{20} \mathrm{H}_{25} \mathrm{FO}_{4}[\mathrm{M}+\mathrm{H}]^{+}$ 347.1653 , found 347.1650 .

( $2 S, 3 R, 6 R$ )-2-甲基-6-((Z)-4-甲基-1-(4-氟苯基)-2-戊 烯-1-酮)-3,6-二氢-2 $H$-吡喃-3-基乙酸酯(3r): 黄色油状, $145 \mathrm{mg}$, 产率 84\%. ${ }^{1} \mathrm{H}$ NMR $\left(\mathrm{CDCl}_{3}, 400 \mathrm{MHz}\right) \delta: 7.82 \sim$ $7.77(\mathrm{~m}, 2 \mathrm{H}), 7.15 \sim 7.09(\mathrm{~m}, 2 \mathrm{H}), 6.10 \sim 6.04(\mathrm{~m}, 2 \mathrm{H})$, 5.89 (ddd, $J=10.0,4.4,2.4 \mathrm{~Hz}, 1 \mathrm{H}), 5.34 \sim 5.32(\mathrm{~m}, 1 \mathrm{H})$, $4.87 \sim 4.85(\mathrm{~m}, 1 \mathrm{H}), 4.11(\mathrm{qd}, J=6.7,2.9 \mathrm{~Hz}, 1 \mathrm{H})$, $3.42 \sim 3.32(\mathrm{~m}, 1 \mathrm{H}), 2.07(\mathrm{~s}, 3 \mathrm{H}), 1.19(\mathrm{~d}, J=6.8 \mathrm{~Hz}, 3 \mathrm{H})$, $1.08(\mathrm{~d}, J=6.6 \mathrm{~Hz}, 3 \mathrm{H}), 0.99(\mathrm{~d}, J=6.6 \mathrm{~Hz}, 3 \mathrm{H}) ;{ }^{13} \mathrm{C}$ NMR $\left(\mathrm{CDCl}_{3}, 100 \mathrm{MHz}\right) \delta: 196.6,171.1,165.5\left(\mathrm{~d}, J_{\mathrm{C}-\mathrm{F}}=\right.$ $252.0 \mathrm{~Hz}), 155.7,136.0,134.4\left(\mathrm{~d}, J_{\mathrm{C}^{-\mathrm{F}}}=3.0 \mathrm{~Hz}\right), 133.9$, $132.6\left(\mathrm{~d}, J_{\mathrm{C}-\mathrm{F}}=9.0 \mathrm{~Hz}\right), 121.1,115.6\left(\mathrm{~d}, J_{\mathrm{C}-\mathrm{F}}=22.0 \mathrm{~Hz}\right)$, 71.0, 68.7, 66.8, 27.8, 22.8, 22.5, 21.5, 16.3. HRMS calcd for $\mathrm{C}_{20} \mathrm{H}_{23} \mathrm{FO}_{4} \mathrm{Na}[\mathrm{M}+\mathrm{Na}]^{+}$369.1473, found 369.1478.

( $2 S, 3 R, 6 R)$-2- 甲基 -6-((Z)-4- 甲基-1-(3,5-二氟苯 基)-2-戊烯-1-酮)-3,6-二氢- $2 H$-吡喃-3-基乙酸酯(3s): 无 色油状, $168 \mathrm{mg}$, 产率 92\%. ${ }^{1} \mathrm{H} \mathrm{NMR}\left(\mathrm{CDCl}_{3}, 400 \mathrm{MHz}\right)$ $\delta: 7.46 \sim 7.40(\mathrm{~m}, 2 \mathrm{H}), 7.06 \sim 7.01(\mathrm{~m}, 1 \mathrm{H}), 5.98 \sim 5.89$ $(\mathrm{m}, 2 \mathrm{H}), 5.70(\mathrm{dd}, J=10.7,1.2 \mathrm{~Hz}, 1 \mathrm{H}), 5.00 \sim 4.96(\mathrm{~m}$, $2 \mathrm{H}), 3.80 \sim 3.74(\mathrm{~m}, 1 \mathrm{H}), 2.22 \sim 2.16(\mathrm{~m}, 1 \mathrm{H}), 2.08(\mathrm{~s}$, $3 \mathrm{H}), 1.13(\mathrm{~d}, J=6.4 \mathrm{~Hz}, 3 \mathrm{H}), 0.96$ (d, $J=6.5 \mathrm{~Hz}, 3 \mathrm{H}), 0.92$ $(\mathrm{d}, J=6.6 \mathrm{~Hz}, 3 \mathrm{H}) ;{ }^{13} \mathrm{C} \mathrm{NMR}\left(\mathrm{CDCl}_{3}, 100 \mathrm{MHz}\right) \delta: 196.1$, $170.5,163.1\left(\mathrm{dd}, J_{\mathrm{C}^{-\mathrm{F}}}=249.0,10.0 \mathrm{~Hz}\right), 140.7\left(\mathrm{t}, J_{\mathrm{C}^{-} \mathrm{F}}=\right.$ $7.0 \mathrm{~Hz}), 135.6,129.6,126.7,115.3\left(\mathrm{dd}, J_{\mathrm{C}-\mathrm{F}}=19.0,7.0\right.$ $\mathrm{Hz}), 111.95,108.6\left(\mathrm{t}, J_{\mathrm{C}-\mathrm{F}}=26.0 \mathrm{~Hz}\right), 72.7,70.0,67.1$, 29.2, 22.6, 22.3, 21.1, 18.2, 17.3. HRMS calcd for $\mathrm{C}_{20} \mathrm{H}_{23} \mathrm{~F}_{2} \mathrm{O}_{4}[\mathrm{M}+\mathrm{H}]^{+}$365.1559, found 365.1567 .

( $2 S, 3 R, 6 R)$-2-甲基-6-((Z)-4-甲基-1-(4-氟苯基)-2-己 烯-1-酮)-3,6-二氢-2 $H$-吡喃-3-基乙酸酯(3t): 无色油状, $148 \mathrm{mg}$, 产率 82\%. ${ }^{1} \mathrm{H} \mathrm{NMR}\left(\mathrm{CDCl}_{3}, 400 \mathrm{MHz}\right) \delta: 7.82 \sim$ $7.77(\mathrm{~m}, 2 \mathrm{H}), 7.14 \sim 7.09(\mathrm{~m}, 2 \mathrm{H}), 6.35(\mathrm{t}, J=7.6 \mathrm{~Hz}, 1 \mathrm{H})$, 6.04 (dd, $J=10.3,1.2 \mathrm{~Hz}, 1 \mathrm{H}), 5.89$ (ddd, $J=10.0,4.4,2.2$ $\mathrm{Hz}, 1 \mathrm{H}), 5.35 \sim 5.33(\mathrm{~m}, 1 \mathrm{H}), 4.88 \sim 4.85(\mathrm{~m}, 1 \mathrm{H}), 4.13$ (qd, $J=6.8,2.8 \mathrm{~Hz}, 1 \mathrm{H}), 2.47 \sim 2.44(\mathrm{~m}, 2 \mathrm{H}), 2.08(\mathrm{~s}, 3 \mathrm{H})$, $1.76 \sim 1.66(\mathrm{~m}, 1 \mathrm{H}), 1.31(\mathrm{~d}, J=6.8 \mathrm{~Hz}, 3 \mathrm{H}), 0.95(\mathrm{~d}, J=$ $6.7 \mathrm{~Hz}, 3 \mathrm{H}), 0.92(\mathrm{~d}, J=6.6 \mathrm{~Hz}, 3 \mathrm{H}) ;{ }^{13} \mathrm{C} \mathrm{NMR}\left(\mathrm{CDCl}_{3}\right.$, $100 \mathrm{MHz}) \delta: 196.1,170.8,165.2\left(\mathrm{~d}, J_{\mathrm{C}-\mathrm{F}}=253.0 \mathrm{~Hz}\right)$, $148.4,138.8,134.2\left(\mathrm{~d}, J_{\mathrm{C}-\mathrm{F}}=3.0 \mathrm{~Hz}\right), 133.5,132.2(\mathrm{~d}$, $\left.J_{\mathrm{C}-\mathrm{F}}=9.0 \mathrm{~Hz}\right), 120.9,115.3\left(\mathrm{~d}, J_{\mathrm{C}^{-} \mathrm{F}}=22.0 \mathrm{~Hz}\right), 71.0,68.4$, 
66.2, 37.3, 28.7, 22.7, 22.6, 21.2, 16.0. HRMS calcd for $\mathrm{C}_{21} \mathrm{H}_{25} \mathrm{FO}_{4} \mathrm{Na}[\mathrm{M}+\mathrm{Na}]^{+}$383.1629, found 383.1628.

(2S,3R,6R)-2- 甲基 -6-(( $Z$ )-1-(3- 基噻吩 )-2-已烯 -1酮)-3,6-二氢- $2 H$-吡喃-3-基乙酸酯 $(3 \mathbf{u})$ : 黄色油状, 114 $\mathrm{mg}$, 产率 68\%. ${ }^{1} \mathrm{H} \mathrm{NMR}\left(\mathrm{CDCl}_{3}, 400 \mathrm{MHz}\right) \delta: 8.01$ (dd, $J=2.9,1.2 \mathrm{~Hz}, 1 \mathrm{H}), 7.55$ (dd, $J=5.1,1.2 \mathrm{~Hz}, 1 \mathrm{H}), 7.33$ (dd, $J=5.1,3.0 \mathrm{~Hz}, 1 \mathrm{H}), 5.97$ (ddd, $J=10.3,2.9,1.6 \mathrm{~Hz}$, $1 \mathrm{H}), 5.88$ (dt, $J=10.3,2.1 \mathrm{~Hz}, 1 \mathrm{H}), 5.85 \sim 5.81(\mathrm{~m}, 1 \mathrm{H})$, 5.06 (br, $1 \mathrm{H}), 5.00(\mathrm{dq}, J=7.5,2.2 \mathrm{~Hz}, 1 \mathrm{H}), 3.83 \sim 3.76$ $(\mathrm{m}, 1 \mathrm{H}), 2.08(\mathrm{~s}, 3 \mathrm{H}) 2.02 \sim 1.92(\mathrm{~m}, 2 \mathrm{H}), 1.44 \sim 1.34(\mathrm{~m}$, $2 \mathrm{H}), 1.10(\mathrm{~d}, J=6.4 \mathrm{~Hz}, 3 \mathrm{H}), 0.84$ (t, $J=7.4 \mathrm{~Hz}, 3 \mathrm{H}) ;{ }^{13} \mathrm{C}$ NMR $\left(\mathrm{CDCl}_{3}, 100 \mathrm{MHz}\right) \delta: 192.5,170.6,142.9,139.6$, $134.7,134.3,130.0,127.2,126.6,126.5,73.0,70.4,66.9$, $31.8,22.5,21.2,17.5,13.7$. HRMS calcd for $\mathrm{C}_{18} \mathrm{H}_{23} \mathrm{O}_{4} \mathrm{~S}$ $[\mathrm{M}+\mathrm{H}]^{+}$357.1132, found 357.1132.

( $2 S, 3 R, 6 R)$-2-甲基-6-((Z)-2-辛烯-4-酮)-3,6-二氢- $2 H$ 吡喃-3-基乙酸酯(3v): 黄色油状, $113 \mathrm{mg}$, 产率 $81 \%$. ${ }^{1} \mathrm{H}$ NMR $\left(\mathrm{CDCl}_{3}, 400 \mathrm{MHz}\right) \delta: 5.94 \sim 5.83(\mathrm{~m}, 3 \mathrm{H}), 5.00 \sim$ $4.97(\mathrm{~m}, 2 \mathrm{H}), 3.76 \sim 3.70(\mathrm{~m}, 1 \mathrm{H}), 2.71 \sim 2.55(\mathrm{~m}, 2 \mathrm{H})$, $2.08(\mathrm{~s}, 3 \mathrm{H}), 1.85(\mathrm{dd}, J=7.2,1.1 \mathrm{~Hz}, 3 \mathrm{H}), 1.65 \sim 1.57(\mathrm{~m}$, $2 \mathrm{H}), 1.40 \sim 1.31(\mathrm{~m}, 2 \mathrm{H}), 1.18(\mathrm{~d}, J=6.4 \mathrm{~Hz}, 3 \mathrm{H}), 0.92(\mathrm{t}$, $J=7.3 \mathrm{~Hz}, 3 \mathrm{H}) ;{ }^{13} \mathrm{C} \mathrm{NMR}\left(\mathrm{CDCl}_{3}, 100 \mathrm{MHz}\right) \delta: 206.1$, $170.6,141.0,133.2,130.4,126.2,71.7,70.2,66.8,42.3$, 25.7, 22.4, 21.1, 17.6, 15.3, 13.9. HRMS calcd for $\mathrm{C}_{16} \mathrm{H}_{25} \mathrm{O}_{4}[\mathrm{M}+\mathrm{H}]^{+}$281.1747, found 281.1751.

(2S,3R,6R)-2-甲基-6-(( $Z$ )-2-十二烯-4-酮)-3,6-二氢$2 \mathrm{H}$-吡喃-3-基乙酸酯(3w): 无色油状, $124 \mathrm{mg}$, 产率 $74 \%$. ${ }^{1} \mathrm{H}$ NMR $\left(\mathrm{CDCl}_{3}, 400 \mathrm{MHz}\right) \delta: 5.94 \sim 5.81(\mathrm{~m}, 3 \mathrm{H})$, $5.00 \sim 4.97(\mathrm{~m}, 2 \mathrm{H}), 3.76 \sim 3.69(\mathrm{~m}, 1 \mathrm{H}), 2.70 \sim 2.54(\mathrm{~m}$, 2H), $2.08(\mathrm{~s}, 3 \mathrm{H}), 1.85(\mathrm{dd}, J=7.2,1.0 \mathrm{~Hz}, 3 \mathrm{H}), 1.66 \sim$ $1.58(\mathrm{~m}, 2 \mathrm{H}), 1.32 \sim 1.24(\mathrm{~m}, 10 \mathrm{H}), 1.18(\mathrm{~d}, J=6.4 \mathrm{~Hz}$, $3 \mathrm{H}), 0.89 \sim 0.86(\mathrm{~m}, 3 \mathrm{H}) ;{ }^{13} \mathrm{C} \mathrm{NMR}\left(\mathrm{CDCl}_{3}, 100 \mathrm{MHz}\right) \delta$ : 206.1, 170.5, 141.0, 133.2, 130.4, 126.2, 71.7, 70.3, 66.7, 42.6, 31.8, 29.4, 29.3, 29.2, 23.6, 22.6, 21.1, 17.6, 15.3, 14.1. HRMS calcd for $\mathrm{C}_{20} \mathrm{H}_{33} \mathrm{O}_{4}[\mathrm{M}+\mathrm{H}]^{+}$337.2373, found 337.2378 .

(2S,3R,6R)-2-甲基-6-(( $Z$ )-2-甲基-3-壬烯-5-酮)-3,6二氢- $2 H$-吡喃-3-基乙酸酯(3x): 黄色油状, $117 \mathrm{mg}$, 产率 $76 \% .{ }^{1} \mathrm{H}$ NMR $\left(\mathrm{CDCl}_{3}, 400 \mathrm{MHz}\right) \delta: 5.94 \sim 5.86(\mathrm{~m}, 2 \mathrm{H})$, $5.47(\mathrm{dd}, J=10.2,1.1 \mathrm{~Hz}, 1 \mathrm{H}), 5.00 \sim 4.94(\mathrm{~m}, 2 \mathrm{H})$, $3.75 \sim 3.69(\mathrm{~m}, 1 \mathrm{H}), 2.70 \sim 2.53(\mathrm{~m}, 3 \mathrm{H}), 2.08(\mathrm{~s}, 3 \mathrm{H})$, $1.64 \sim 1.56(\mathrm{~m}, 3 \mathrm{H}), 1.39 \sim 1.32(\mathrm{~m}, 2 \mathrm{H}), 1.18(\mathrm{~d}, J=6.4$ $\mathrm{Hz}, 3 \mathrm{H}), 1.05$ (d, $J=6.6 \mathrm{~Hz}, 3 \mathrm{H}), 0.97$ (d, $J=6.6 \mathrm{~Hz}, 3 \mathrm{H})$, $0.92(\mathrm{t}, J=7.3 \mathrm{~Hz}, 3 \mathrm{H}) ;{ }^{13} \mathrm{C} \mathrm{NMR}\left(\mathrm{CDCl}_{3}, 100 \mathrm{MHz}\right) \delta$ : 206.8, 170.9, 144.5, 138.5, 130.7, 126.5, 72.2, 70.5, 67.0,
43.0, 28.9, 26.0, 23.3, 23.2, 22.7, 21.4, 17.9, 14.2. HRMS calcd for $\mathrm{C}_{18} \mathrm{H}_{29} \mathrm{O}_{4}[\mathrm{M}+\mathrm{H}]^{+}$309.2060, found 309.2063.

( $2 S, 3 R, 6 R)$-2-甲基-6-( $(Z)-1$-(4-甲基苯基)-1-庚烯-3酮)-3,6-二氢- $2 H$-吡喃-3-基乙酸酯(3y): 黄色油状, 116 $\mathrm{mg}$, 产率 65\%. ${ }^{1} \mathrm{H} \mathrm{NMR}\left(\mathrm{CDCl}_{3}, 400 \mathrm{MHz}\right) \delta: 7.14 \sim 7.09$ (m, 4H), 6.69 (s, 1H), 6.01 (ddd, $J=10.3,2.9,1.5 \mathrm{~Hz}, 1 \mathrm{H})$, 5.95 (dt, $J=10.3,2.0 \mathrm{~Hz}, 1 \mathrm{H}), 5.14 \sim 5.12(\mathrm{~m}, 1 \mathrm{H}), 5.04$ $(\mathrm{dq}, J=7.2,2.4 \mathrm{~Hz}, 1 \mathrm{H}), 3.88 \sim 3.81(\mathrm{~m}, 1 \mathrm{H}), 2.35(\mathrm{~s}, 3 \mathrm{H})$, $2.33 \sim 2.23(\mathrm{~m}, 2 \mathrm{H}), 2.12(\mathrm{~s}, 3 \mathrm{H}), 1.59 \sim 1.55(\mathrm{~m}, 2 \mathrm{H})$, $1.54 \sim 1.42(\mathrm{~m}, 2 \mathrm{H}), 1.22(\mathrm{~d}, J=6.3 \mathrm{~Hz}, 3 \mathrm{H}), 0.79(\mathrm{t}, J=$ $7.3 \mathrm{~Hz}, 3 \mathrm{H}) ;{ }^{13} \mathrm{C} \mathrm{NMR}\left(\mathrm{CDCl}_{3}, 100 \mathrm{MHz}\right) \delta: 209.4,170.5$, $141.3,138.6,132.4,132.4,130.2,129.2,128.6,126.6$, 73.1, 70.4, 67.0, 43.3, 25.7, 22.0, 21.3, 21.2, 17.7, 13.8 . HRMS calcd for $\mathrm{C}_{22} \mathrm{H}_{29} \mathrm{O}_{4}[\mathrm{M}+\mathrm{H}]^{+} 357.2060$, found 357.2066 .

\section{2 .2 化合物 4 的合成}

在 $25 \mathrm{~mL}$ 的 Schlenk 管内, 依次加入 $L$-鼠李烯糖 $\mathbf{1}$ (0.5 mmol), 炔丙酯 2 (0.5 mmol), $\mathrm{CH}_{3} \mathrm{NO}_{2}(5 \mathrm{~mL})$, 室温 搅拌 $10 \mathrm{~min}$ 后, 迅速加入 $\mathrm{Ph}_{3} \mathrm{PAuCl}(12.3 \mathrm{mg}, 5 \mathrm{~mol} \%)$ 和 $\mathrm{AgSbF}_{6}(6.5 \mathrm{mg}, 5 \mathrm{~mol} \%)$. 室温下搅拌 $6 \mathrm{~h}, \mathrm{TLC}$ 检测 反应完全. 用砂芯漏斗过滤, 减压浓缩除去溶剂. 硅胶 快速色谱法(石油醚/乙酸乙酯, $V: V=10: 1$ )纯化残余 物, 得到 $C$-乙烯基鼠李糖苷衍生物 4 .

( $2 S, 3 R, 6 R)$-2-甲基-6-(( $Z$ )-1-苯基-2-戊烯-1-酮)-3,6二氢- $2 H$-吡喃-3-苯基乙酸酯(4ba): 无色油状, $143 \mathrm{mg}$, 产率 76\%. ${ }^{1} \mathrm{H}$ NMR $\left(\mathrm{CDCl}_{3}, 400 \mathrm{MHz}\right) \delta: 8.05 \sim 8.03(\mathrm{~m}$, 2H), $7.97 \sim 7.94(\mathrm{~m}, 2 \mathrm{H}), 7.61 \sim 7.56(\mathrm{~m}, 2 \mathrm{H}), 7.51 \sim 7.44$ $(\mathrm{m}, 4 \mathrm{H}), 6.04 \sim 6.03(\mathrm{~m}, 1 \mathrm{H}), 5.92(\mathrm{td}, J=7.7,1.2 \mathrm{~Hz}$, $1 \mathrm{H}), 5.27 \sim 5.24(\mathrm{~m}, 1 \mathrm{H}), 5.13(\mathrm{br}, 1 \mathrm{H}), 4.03 \sim 3.97(\mathrm{~m}$, $1 \mathrm{H}), 3.49$ (d, $J=5.4 \mathrm{~Hz}, 1 \mathrm{H}), 2.05 \sim 1.88(\mathrm{~m}, 2 \mathrm{H}), 1.19(\mathrm{~d}$, $J=6.3 \mathrm{~Hz}, 3 \mathrm{H}), 0.96(\mathrm{t}, J=7.4 \mathrm{~Hz}, 3 \mathrm{H}) ;{ }^{13} \mathrm{C} \mathrm{NMR}\left(\mathrm{CDCl}_{3}\right.$, $100 \mathrm{MHz}) \delta$ : 198.8, 166.1, 137.9, 137.5, 136.7, 133.5, $133.2,130.3,130.1,129.7,129.3,128.7,128.4,126.5$, 73.1, 70.8, 67.2, 23.3, 17.6, 13.7. HRMS calcd for $\mathrm{C}_{24} \mathrm{H}_{24} \mathrm{O}_{4} \mathrm{Na}[\mathrm{M}+\mathrm{Na}]^{+}$399.1567, found 399.1566.

( $2 S, 3 R, 6 R)$-2-甲基-6-(( $Z$ )-5-甲基-1-苯基-2-己烯-1酮)-3,6-二氢-2H-吡喃-3-苯基乙酸酯(4bb): 无色油状, $148 \mathrm{mg}$, 产率 $73 \%$. ${ }^{1} \mathrm{H} \mathrm{NMR}\left(\mathrm{CDCl}_{3}, 400 \mathrm{MHz}\right) \delta: 8.05$ $8.02(\mathrm{~m}, 2 \mathrm{H}), 7.96 \sim 7.93(\mathrm{~m}, 2 \mathrm{H}), 7.61 \sim 7.56(\mathrm{~m}, 2 \mathrm{H})$, $7.50 \sim 7.43(\mathrm{~m}, 4 \mathrm{H}), 6.07 \sim 6.03(\mathrm{~m}, 2 \mathrm{H}), 5.97(\mathrm{td}, J=7.0$, $1.2 \mathrm{~Hz}, 1 \mathrm{H}), 5.27 \sim 5.24(\mathrm{~m}, 1 \mathrm{H}), 5.13(\mathrm{br}, 1 \mathrm{H}), 4.03 \sim$ $3.97(\mathrm{~m}, 1 \mathrm{H}), 1.93 \sim 1.77(\mathrm{~m}, 2 \mathrm{H}), 1.72 \sim 1.65(\mathrm{~m}, 1 \mathrm{H})$, $1.19(\mathrm{~d}, J=6.3 \mathrm{~Hz}, 3 \mathrm{H}), 0.84(\mathrm{t}, J=6.9 \mathrm{~Hz}, 6 \mathrm{H}) ;{ }^{13} \mathrm{C} \mathrm{NMR}$ $\left(\mathrm{CDCl}_{3}, 100 \mathrm{MHz}\right) \delta: 198.9,166.1,139.2,137.4,134.4$, $133.4,133.2$, 130.3, 130.1, 129.7, 129.3, 128.7, 128.4, 
126.5, 73.2, 70.8, 67.1, 38.5, 28.6, 22.4, 22.2, 17.6. HRMS calcd for $\mathrm{C}_{26} \mathrm{H}_{28} \mathrm{O}_{4} \mathrm{Na}[\mathrm{M}+\mathrm{Na}]^{+}$427.1880, found 427.1881 .

( $2 S, 3 R, 6 R)$-2- 甲基-6-(( $Z$ )-5-甲基-1-苯基-2-己烯-1酮)-3,6-二氢- $2 H$-吡喃-3-(2-氯苯基)-乙酸酯(4ca): 无色 油状, $162 \mathrm{mg}$, 产率 $74 \% .{ }^{1} \mathrm{H} \mathrm{NMR}\left(\mathrm{CDCl}_{3}, 400 \mathrm{MHz}\right) \delta$ : $7.93 \sim 7.91(\mathrm{~m}, 2 \mathrm{H}), 7.78(\mathrm{dd}, J=7.7,1.5 \mathrm{~Hz}, 1 \mathrm{H}), 7.60 \sim$ $7.55(\mathrm{~m}, 1 \mathrm{H}), 7.49 \sim 7.41(\mathrm{~m}, 4 \mathrm{H}), 7.35 \sim 7.29(\mathrm{~m}, 1 \mathrm{H})$, $6.08 \sim 6.03(\mathrm{~m}, 2 \mathrm{H}), 5.95(\mathrm{td}, J=7.1,1.3 \mathrm{~Hz}, 1 \mathrm{H}), 5.28 \sim$ $5.26(\mathrm{~m}, 1 \mathrm{H}), 5.13(\mathrm{br}, 1 \mathrm{H}), 4.04 \sim 3.97(\mathrm{~m}, 1 \mathrm{H}), 1.90 \sim$ $1.75(\mathrm{~m}, 2 \mathrm{H}), 1.69 \sim 1.63(\mathrm{~m}, 1 \mathrm{H}), 1.22(\mathrm{~d}, J=6.3 \mathrm{~Hz}$, $3 \mathrm{H}), 0.82(\mathrm{t}, J=6.4 \mathrm{~Hz}, 6 \mathrm{H}) ;{ }^{13} \mathrm{C} \mathrm{NMR}\left(\mathrm{CDCl}_{3}, 100 \mathrm{MHz}\right)$ $\delta: 198.8,165.4,139.1,137.4,134.5,133.7,133.4,132.7$, $131.4,131.1,130.7,130.1,129.3,128.7,126.6,126.0$, 73.1, 71.5, 67.0, 38.5, 28.5, 22.4, 22.2, 17.7. HRMS calcd for $\mathrm{C}_{26} \mathrm{H}_{27} \mathrm{ClO}_{4} \mathrm{Na}[\mathrm{M}+\mathrm{Na}]^{+}$461.1490, found 461.1491.

( $2 S, 3 R, 6 R)$-2-甲基-6-( $Z$ )-5-甲基-1-苯基-2-己烯-1酮)-3,6-二氢- $2 H$-吡喃-3-(3-氯苯基)-乙酸酯(4da): 无色 油状, $164 \mathrm{mg}$, 产率 75\%. ${ }^{1} \mathrm{H} \mathrm{NMR}\left(\mathrm{CDCl}_{3}, 400 \mathrm{MHz}\right) \delta$ : $8.04(\mathrm{t}, J=1.9 \mathrm{~Hz}, 1 \mathrm{H}), 7.95(\mathrm{dt}, J=7.8,1.4 \mathrm{~Hz}, 1 \mathrm{H})$, $7.78 \sim 7.76(\mathrm{~m}, 2 \mathrm{H}), 7.56 \sim 7.52(\mathrm{~m}, 2 \mathrm{H}), 7.46 \sim 7.42(\mathrm{~m}$, 2H), 7.37 (t, $J=7.9 \mathrm{~Hz}, 1 \mathrm{H}), 6.42$ (t, $J=7.4 \mathrm{~Hz}, 1 \mathrm{H}), 6.15$ (dd, $J=10.2,1.8 \mathrm{~Hz} 1 \mathrm{H}$ ), 6.01 (ddd, $J=10.2,4.7,2.5 \mathrm{~Hz}$, $1 \mathrm{H}), 5.46 \sim 5.44(\mathrm{~m}, 1 \mathrm{H}), 5.12 \sim 5.10(\mathrm{~m}, 1 \mathrm{H}), 4.31(\mathrm{qd}$, $J=7.1,2.4 \mathrm{~Hz}, 1 \mathrm{H}), 2.57 \sim 2.44(\mathrm{~m}, 2 \mathrm{H}), 1.76 \sim 1.69(\mathrm{~m}$, $1 \mathrm{H}), 1.39$ (d, $J=6.8 \mathrm{~Hz}, 3 \mathrm{H}), 0.88(\mathrm{t}, J=7.2 \mathrm{~Hz}, 6 \mathrm{H}) ;{ }^{13} \mathrm{C}$ NMR $\left(\mathrm{CDCl}_{3}, 100 \mathrm{MHz}\right) \delta: 197.6,165.2,149.2,138.7$, $138.0,134.5,133.1,132.2,131.9,129.8,129.7,129.7$, 128.2, 128.0, 120.4, 71.2, 69.4, 66.0, 37.3, 28.7, 22.6, 22.5, 16.0. HRMS calcd for $\mathrm{C}_{26} \mathrm{H}_{27} \mathrm{ClO}_{4} \mathrm{Na}\left[\mathrm{M}+\mathrm{Na}{ }^{+}\right.$ 461.1490, found 461.1491.

$(2 S, 3 R, 6 R)-2$ - 甲基-6-(( $Z$ )-5-甲基-1-苯基-2-己烯-1酮)-3,6-二氢- $2 H$-吡喃-3-(2-氟苯基)-乙酸酯(4ea): 无色 油状, $160 \mathrm{mg}$, 产率 $76 \% .{ }^{1} \mathrm{H} \mathrm{NMR}\left(\mathrm{CDCl}_{3}, 400 \mathrm{MHz}\right) \delta$ : $7.94 \sim 7.89(\mathrm{~m}, 3 \mathrm{H}), 7.60 \sim 7.53(\mathrm{~m}, 2 \mathrm{H}), 7.50 \sim 7.49(\mathrm{~m}$, $2 \mathrm{H}), 7.22 \sim 7.13(\mathrm{~m}, 2 \mathrm{H}), 6.07 \sim 6.01(\mathrm{~m}, 2 \mathrm{H}), 5.96(\mathrm{td}$, $J=7.0,1.2 \mathrm{~Hz}, 1 \mathrm{H}), 5.27 \sim 5.24(\mathrm{~m}, 1 \mathrm{H}), 5.13$ (br, $1 \mathrm{H})$, $4.03 \sim 3.97(\mathrm{~m}, 1 \mathrm{H}), 1.92 \sim 1.75(\mathrm{~m}, 2 \mathrm{H}), 1.69 \sim 1.62(\mathrm{~m}$, $1 \mathrm{H}), 1.20(\mathrm{~d}, J=6.4 \mathrm{~Hz}, 3 \mathrm{H}), 0.83(\mathrm{~m}, J=6.4 \mathrm{~Hz}, 6 \mathrm{H}) ;{ }^{13} \mathrm{C}$ NMR $\left(\mathrm{CDCl}_{3}, 100 \mathrm{MHz}\right) \delta: 198.9,164.0,164.0,162.0$ (d, $\left.J_{\mathrm{C}-\mathrm{F}}=259.0 \mathrm{~Hz}\right), 139.1,137.4,134.7\left(\mathrm{~d}, J_{\mathrm{C}-\mathrm{F}}=9.0 \mathrm{~Hz}\right)$, $134.5,133.4,132.1,130.4,129.3,128.7,126.4,124.0$ (d, $\left.J_{\mathrm{C}^{-\mathrm{F}}}=4.0 \mathrm{~Hz}\right), 118.8,118.7,117.0\left(\mathrm{~d}, J_{\mathrm{C}^{-\mathrm{F}}}=22.0 \mathrm{~Hz}\right)$, 73.2, 71.4, 66.9, 38.5, 28.6, 22.4, 22.2, 17.6. HRMS calcd for $\mathrm{C}_{26} \mathrm{H}_{27} \mathrm{FO}_{4} \mathrm{Na}[\mathrm{M}+\mathrm{Na}]^{+}$445.1786, found 445.1784.
(2S,3R,6R)-2-甲基-6-(( $Z$ )-5-甲基-1-苯基-2-己烯-1酮)-3,6-二氢- $2 H$-吡喃-3-(4-氟苯基)-乙酸酯(4fa): 无色 油状, $165 \mathrm{mg}$, 产率 $78 \% .{ }^{1} \mathrm{H} \mathrm{NMR}\left(\mathrm{CDCl}_{3}, 400 \mathrm{MHz}\right) \delta$ : $8.09 \sim 8.06(\mathrm{~m}, 2 \mathrm{H}), 7.78 \sim 7.76(\mathrm{~m}, 2 \mathrm{H}), 7.56 \sim 7.52(\mathrm{~m}$, $1 \mathrm{H}), 7.46 \sim 7.42(\mathrm{~m}, 2 \mathrm{H}), 7.12 \sim 7.6(\mathrm{~m}, 2 \mathrm{H}), 6.40(\mathrm{t}, J=$ $7.4 \mathrm{~Hz}, 1 \mathrm{H}), 6.14(\mathrm{dd}, J=10.3,1.9 \mathrm{~Hz}, 1 \mathrm{H}), 6.01$ (ddd, $J=$ $10.3,4.8,2.5 \mathrm{~Hz}, 1 \mathrm{H}), 5.45 \sim 5.44(\mathrm{~m}, 1 \mathrm{H}), 5.11 \sim 5.09(\mathrm{~m}$, $1 \mathrm{H}), 4.30(\mathrm{qd}, J=6.8,2.4 \mathrm{~Hz}, 1 \mathrm{H}), 2.57 \sim 2.43(\mathrm{~m}, 2 \mathrm{H})$, $1.75 \sim 1.66(\mathrm{~m}, 1 \mathrm{H}), 1.39(\mathrm{~d}, J=6.8 \mathrm{~Hz}, 3 \mathrm{H}), 0.88(\mathrm{t}, J=$ $6.8 \mathrm{~Hz}, 6 \mathrm{H}) ;{ }^{13} \mathrm{C} \mathrm{NMR}\left(\mathrm{CDCl}_{3}, 100 \mathrm{MHz}\right) \delta: 197.6,165.8$ $\left(\mathrm{d}, J_{\mathrm{C}-\mathrm{F}}=253.0 \mathrm{~Hz}\right), 165.4,148.8,138.8,138.1,134.2$, $132.4\left(\mathrm{~d}, J_{\mathrm{C}-\mathrm{F}}=9.0 \mathrm{~Hz}\right), 132.2,129.7,128.2,126.3(\mathrm{~d}$, $\left.J_{\mathrm{C}-\mathrm{F}}=3.0 \mathrm{~Hz}\right), 120.7,115.5\left(\mathrm{~d}, J_{\mathrm{C}-\mathrm{F}}=22.0 \mathrm{~Hz}\right), 71.2,69.1$, $66.0,37.3,28.7,22.6,22.5,16.1$. HRMS calcd for $\mathrm{C}_{26} \mathrm{H}_{27^{-}}$ $\mathrm{FO}_{4} \mathrm{Na}[\mathrm{M}+\mathrm{Na}]^{+}$445.1786, found 445.1784.

\section{2 .3 化合物 $\mathbf{5 a} \sim \mathbf{5 b}$ 的合成}

在 $25 \mathrm{~mL}$ 的 Schlenk 管内, 依次加入 $L$-岩藻烯糖 $\mathbf{1 g}$ (107 mg, $0.5 \mathrm{mmol})$, 炔丙酯 $2(0.5 \mathrm{mmol})$ 以及 $\mathrm{CH}_{3} \mathrm{NO}_{2}$ $(5 \mathrm{~mL})$. 室温搅拌 $10 \mathrm{~min}$ 后, 迅速加入 $\mathrm{Ph}_{3} \mathrm{PAuCl}(12.3$ $\mathrm{mg}, 5 \mathrm{~mol} \%)$ 和 $\mathrm{AgSbF}_{6}(6.5 \mathrm{mg}, 5 \mathrm{~mol} \%)$. 室温下搅拌 6 $h$, TLC 检测反应完全. 用砂芯漏斗过滤，减压浓缩除去 溶剂. 硅胶快速色谱法(PE：EtOAc, $V: V=20: 1)$ 纯化 残余物, 得到 $C$-乙烯基岩藻糖苷衍生物 $\mathbf{5}$.

(2S,3S,6R)-2-甲基-6-((Z)-1-苯基-2-戊烯-1-酮)-3,6二氢- $2 H$-吡喃-3-基乙酸酯(5a): 无色油状, $151 \mathrm{mg}$, 产率 96\%. ${ }^{1} \mathrm{H}$ NMR $\left(\mathrm{CDCl}_{3}, 400 \mathrm{MHz}\right) \delta: 7.91 \sim 7.88(\mathrm{~m}, 2 \mathrm{H})$, $7.61 \sim 7.57(\mathrm{~m}, 1 \mathrm{H}), 7.50 \sim 7.46(\mathrm{~m}, 2 \mathrm{H}), 6.17 \sim 6.07(\mathrm{~m}$, $2 \mathrm{H}), 5.80(\mathrm{td}, J=7.7,1.3 \mathrm{~Hz}, 1 \mathrm{H}), 5.20 \sim 5.19(\mathrm{~m}, 1 \mathrm{H})$, $5.00(\mathrm{dd}, J=4.8,2.4 \mathrm{~Hz}, 1 \mathrm{H}), 4.06(\mathrm{qd}, J=6.5,2.4 \mathrm{~Hz}$, $1 \mathrm{H}), 2.09$ (s, 3H), $1.98 \sim 1.90(\mathrm{~m}, 2 \mathrm{H}) 1.08(\mathrm{~d}, J=6.6 \mathrm{~Hz}$, $3 \mathrm{H}), 0.94$ (t, $J=7.4 \mathrm{~Hz}, 3 \mathrm{H}) ;{ }^{13} \mathrm{C} \mathrm{NMR}\left(\mathrm{CDCl}_{3}, 100 \mathrm{MHz}\right)$ $\delta: 198.7,170.9,137.5,137.4,136.7,133.4,132.1,129.1$, $128.7,124.7,73.7,66.2,66.0,23.2,20.9,16.0,13.7$. HRMS calcd for $\mathrm{C}_{19} \mathrm{H}_{22} \mathrm{O}_{4} \mathrm{Na}[\mathrm{M}+\mathrm{Na}]^{+} 337.1410$, found 337.1412 .

( $2 S, 3 S, 6 R)$-2-甲基-6-(( $Z$ )-5-甲基-1-(4-丁基苯基)-2己烯-1-酮)-3,6-二氢- $2 H$-吡喃-3-基乙酸酯(5b): 无色油 状, $183 \mathrm{mg}$, 产率 92\%. ${ }^{1} \mathrm{H} \mathrm{NMR}\left(\mathrm{CDCl}_{3}, 400 \mathrm{MHz}\right) \delta$ : $7.73 \sim 7.71(\mathrm{~m}, 2 \mathrm{H}), 7.26 \sim 7.24(\mathrm{~m}, 2 \mathrm{H}), 6.17(\mathrm{t}, J=7.5$ $\mathrm{Hz}, 1 \mathrm{H}), 6.09$ (dd, $J=10.1,2.9 \mathrm{~Hz}, 1 \mathrm{H}), 5.95$ (ddd, $J=$ 10.2, 4.6, $2.4 \mathrm{~Hz}, 1 \mathrm{H}), 5.39$ (br, 1H), $5.14 \sim 5.12(\mathrm{~m}, 1 \mathrm{H})$, $4.22(\mathrm{qd}, J=6.6,3.1 \mathrm{~Hz}, 1 \mathrm{H}), 2.67$ (t, $J=7.7 \mathrm{~Hz}, 2 \mathrm{H}), 2.34$ $(\mathrm{t}, J=7.2 \mathrm{~Hz}, 2 \mathrm{H}), 1.77 \sim 1.70(\mathrm{~m}, 1 \mathrm{H}), 1.66 \sim 1.60(\mathrm{~m}$, $2 \mathrm{H}), 1.42 \sim 1.32(\mathrm{~m}, 2 \mathrm{H}), 1.16(\mathrm{~d}, J=6.5 \mathrm{~Hz}, 3 \mathrm{H}), 0.96 \sim$ 0.92 (m, 9H); ${ }^{13} \mathrm{C} \mathrm{NMR}\left(\mathrm{CDCl}_{3}, 100 \mathrm{MHz}\right) \delta: 197.3$, 
$170.8,148.4,144.5,139.5,135.5,132.6,130.1,128.4$, $122.7,69.0,68.0,66.8,37.3,35.7,33.3,29.7,28.6,22.6$, 22.5, 22.4, 21.0, 15.5, 13.9. HRMS calcd for $\mathrm{C}_{25} \mathrm{H}_{34} \mathrm{O}_{4} \mathrm{Na}$ $[\mathrm{M}+\mathrm{Na}]^{+}$421.2349, found 421.2341.

\section{2 .4 化合物 $3 \mathbf{a}^{-18} \mathbf{O}$ 的合成}

在 $25 \mathrm{~mL}$ 的 Schlenk 管内, 依次加入 $L$-鼠李烯糖 $\mathbf{1 a}$ (54 mg, $0.25 \mathrm{mmol}$ ), 炔丙酯 $\mathbf{2 a -}{ }^{\mathbf{1 8}} \mathbf{O}$ (54 mg, $0.25 \mathrm{mmol}$ ), 以及 $\mathrm{CH}_{3} \mathrm{NO}_{2}(3 \mathrm{~mL})$. 室温搅拌 $10 \mathrm{~min}$ 后, 快速加入 $\mathrm{Ph}_{3} \mathrm{PAuCl}(6 \mathrm{mg}, 5 \mathrm{~mol} \%)$ 和 $\mathrm{AgSbF}_{6}$ (3 mg, $5 \mathrm{~mol} \%$ ). 室 温下搅拌 $6 \mathrm{~h}, \mathrm{TLC}$ 检测反应完全. 用砂芯漏斗过滤, 减 压浓缩除去溶剂. 通过硅胶快速色谱法(石油醚/乙酸乙 酯, $V: V=20: 1)$ 纯化残余物, 得到 $C$-乙烯基鼠李糖苷 衍生物 3a- ${ }^{18} \mathbf{O}$.

( $2 S, 3 R, 6 R)$-2- 甲基 -6-(( $Z)-1$ - 苯基 -2- 己烯 -1-( 酮 $\left.\left.{ }^{18} \mathrm{O}\right)\right)-3,6$-二氢- $2 H$-吡喃-3-基乙酸酯 $\left(\mathbf{3 a}-{ }^{18} \mathbf{O}\right)$ : 无色油状, $65 \mathrm{mg}$, 产率 79\%. ${ }^{1} \mathrm{H} \mathrm{NMR}\left(\mathrm{CDCl}_{3}, 400 \mathrm{MHz}\right) \delta: 7.93 \sim$ $7.90(\mathrm{~m}, 2 \mathrm{H}), 7.60 \sim 7.56(\mathrm{~m}, 1 \mathrm{H}), 7.49 \sim 7.46(\mathrm{~m}, 2 \mathrm{H})$, 5.98 (ddd, $J=10.3,2.9,1.6 \mathrm{~Hz}, 1 \mathrm{H}), 5.91 \sim 5.87$ (m, 2H), $5.08(\mathrm{br}, 1 \mathrm{H}), 5.00(\mathrm{dq}, J=7.6,2.2 \mathrm{~Hz}, 1 \mathrm{H}), 3.85 \sim 3.79$ $(\mathrm{m}, 1 \mathrm{H}), 2.09(\mathrm{~s}, 3 \mathrm{H}), 1.95 \sim 1.86(\mathrm{~m}, 2 \mathrm{H}), 1.42 \sim 1.34(\mathrm{~m}$, $2 \mathrm{H}), 1.11(\mathrm{~d}, J=6.3 \mathrm{~Hz}, 3 \mathrm{H}), 0.81(\mathrm{t}, J=7.4 \mathrm{~Hz}, 3 \mathrm{H}) ;{ }^{13} \mathrm{C}$ NMR $\left(\mathrm{CDCl}_{3}, 100 \mathrm{MHz}\right) \delta$ : 198.8, 198.8, 170.5, 138.6, 137.5, 135.2, 133.4, 130.1, 129.2, 128.6, 126.6, 73.1, 70.4, 66.9, 31.7, 29.7, 22.4, 21.2, 17.5, 13.7. HRMS calcd for $\mathrm{C}_{20} \mathrm{H}_{24} \mathrm{O}_{3}{ }^{18} \mathrm{ONa} \quad[\mathrm{M}+\mathrm{Na}]^{+}$353.1609, found 353.1610.

\section{辅助材料(Supporting Information) 化合物 3, 4, 5 的核} 磁谱图, 化合物 3g (CCDC 1965665)的单晶结构数据. 这些材料可以免费从本刊网站(http://sioc-journal.cn/)上 下载.

\section{References}

[1] (a) Compain, P.; Martin, O. R. Bioorg. Med. Chem. 2001, 9, 3077. (b) Chen, C. L.; Sparks, S. M.; Martin, S. F. J. Am. Chem. Soc. 2006, 128, 13696

(c) Štambaský, J.; Hocek, M.; Kocovský, P. Chem. Rev. 2009, 109, 6729

(d) Kitamura, K.; Ando, Y.; Matsumoto, T.; Suzuki, K. Chem. Rev. 2018, 118,1495 .

(e) Liao, H. Z; Ma, J.; Yao, H.; Liu, X. W. Org. Biomol. Chem. 2018, 16, 1791

(f) Norsikian, S.; Tresse, C.; François-Eude, M.; Jeanne-Julien, L.; Masson, G.; Servajean, V.; Genta-Jouve, G.; Beau, J. M.; Roull, E. Angew. Chem., Int. Ed. 2020, 59, 6612.

(g) Kitamura, K.; Ando, Y.; Matsumoto, T.; Suzuki, K. Chem. Rev. 2018, 118, 1495.

[2] (a) Hassanzadeh, A.; Gorry, P. A.; Morris, G. A.; Barber, J. J. Med. Chem. 2006, 49, 6334 .

(b) Kim, B. G.; Kim, H. J.; Ahn, J. H. J. Agric. Food. Chem. 2012, 60,11143

(c) Bhattarai, B.; Nagorny, P. Org. Lett. 2018, 20, 154.

(d) Chauvin, A.; Nepogodiev, S.; Field, R. J. Org. Chem. 2005, 70,
960.

(e) Urabe, D.; Nakagawa, Y.; Mukai, K.; Fukushima, K.; Aoki, N.; Itoh, H.; Nagatomo, M.; Inoue, M. J. Org. Chem. 2018, 83, 13888. (f) Lee, H. S.; Kim, M. J. J. Agric. Food. Chem. 2002, 50, 1840. (g) Khatri, H. R.; Bhattarai, B.; Kaplan, W.; Li, Z.; Long, M. J. C.; Aye, Y.; Nagorny, P. J. Am. Chem. Soc. 2019, 141, 4849.

(h) Crich, D.; Li, H. J. Org. Chem. 2002, 67, 4640.

(i) Ashraf Shalaby, M.; Fronczek, F. R.; Younathan, E. S. Carbohydr. Res. 1994, 258, 267

[3] (a) Chen, C. L.; Sparks, S. M.; Martin, S. F. J. Am. Chem. Soc. 2006, $128,13696$.

(b) Tius, M. A.; Gu, X.; Gomez-Galeno, J. J. Am. Chem. Soc. 1990 112,8188 .

(c) Liao, H. Z; Ma, J.; Yao, H.; Liu, X. W. Org. Biomol. Chem. 2018, 16, 1791.

[4] (a) Cai, X.; Ng, K.; Panesar, H.; Moon, S. J.; Paredes, M.; Ishida, K.; Hertweck, C.; Minehan, T. G. Org. Lett. 2014, 16, 2962. (b) Liao, H. Z; Ma, J.; Yao, H.; Liu, X. W. Org. Biomol. Chem. 2018, 16, 1791.

[5] Szeja, W.; Grynkiewicz, G.; Bieg, T.; Swierk, P.; Byczek, A.; Papaj, K.; Kitel, R.; Rusin, A. Molecules 2014, 19, 7072.

[6] (a) Gong, H.; Sinisi, R.; Gagne, M. R. J. Am. Chem. Soc. 2007, 129, 1908.

(b) Gong, H.; Gagne, M. R. J. Am. Chem. Soc. 2008, $130,12177$.

(c) Andrews, R. S.; Becker, J. J.; Gagné, M. R. Angew. Chem., Int. Ed. 2010, 49, 7274.

(d) Nicolas, L.; Angibaud, P.; Stansfield, I.; Bonnet, P.; Meerpoel, L.; Reymond, S.; Cossy, J. Angew. Chem., Int. Ed. 2012, 51, 11101. (e) Andrews, R. S.; Becker, J. J.; Gagné, M. R. Angew. Chem., Int. Ed. 2012, 51, 4140.

(f) Zhao, C.; Jia, X.; Wang, X.; Gong, H. J. Am. Chem. Soc. 2014, 136, 17645.

(g) Zhu, F.; Rourke, M. J.; Yang, T.; Rodriguez, J.; Walczak, M. A. J. Am. Chem. Soc. 2016, 138, 1204

(h) Adak, L.; Kawamura, S.; Toma, G.; Takenaka, T.; Isozaki, K.; Takaya, H.; Orita, A.; Li, H. C.; Shing, T. K. M.; Nakamura, M. J. Am. Chem. Soc. 2017, 139, 10693.

[7] (a) Koppolu, S. R.; Niddana, R.; Balamurugan, R. Org. Biomol. Chem. 2015, 13, 5094.

(b) Wang, Y.; Liu, M.; Liu, L.; Xia, J. H.; Du, Y. G.; Sun, J. S. J. Org. Chem. 2018, 83, 4111.

[8] (a) Bolitt, V.; Mioskowski, C.; Kollah, R. O.; Manna, S.; Rajapaksa, D.; Falck, J. R. J. Am. Chem. Soc. 1991, 113, 6320.

(b) Fuganti, C.; Serra, S. Synlett 1999, 1999, 1241.

(c) Kulkarni, S. S.; Gervay-Hague, J. Org. Lett. 2006, 8, 5765.

(d) Kaliappan, K. P.; Subrahmanyam, A. V. Org. Lett. 2007, 9, 1121.

(e) Snajdr, I.; Parkan, K.; Hessler, F.; Kotora, M. Beilstein J. Org. Chem. 2015, 11, 1392

[9] (a) Li, X.; Chen, G.; Garcia-Navarro, R.; Franck, R. W.; Tsuji, M. Immunology 2009, 127, 216.

(b) Yao, Y.; Xiong, C. P.; Zhong, y. L.; Bian, G. W.; Huang, N. Y.; Wang, L.; Zou, K. Adv. Synth. Catal. 2019, 51012.

[10] Bai, Y.; Leow, M.; Zeng, J.; Liu, X.-W. Org. Lett. 2011, 13, 5648.

[11] Tatina, M.; Kusunuru, A. K.; Yousuf, S. K.; Mukherjee, D. Chem. Commun. 2013, 49, 11409.

[12] (a) Sharma, B. M.; Rathod, J.; Gonnade, R. G.; Kumar, P. J. Org. Chem. 2018, 83, 9353.

(b) Ruengsangtongkul, S.; Chaisan, N.; Thongsornkleeb, C.; Tummatorn, J. Org. Lett. 2019, 21, 2514.

[13] Veryser, C.; Steurs, G.; Meervelt, L. V.; Borggraeve, W. M. Adv. Synth. Catal. 2017, 359, 1271.

[14] Xiao, Q.; Zheng, F.; Tang, Q.; Wu, J. J.; Xie, J.; Huang, H. D.; Yang, X. B.; Hann, S. S. Cell Physiol. Biochem. 2018, 49, 1615.

[15] (a) Xu, Y.; Wang, W. J.; Cai, Y.; Yang, X.; Wang, P. G.; Zhao, W. RSC Adv. 2014, 4, 46662.

(b) Gagarinov, I. A.; Fang, T.; Liu, L.; Srivastava, A. D.; Boons, G. J. Org. Lett. 2015, 17, 928 . 
(c) Suzuki, K.; Sulikowski, G. A.; Friesen, R. W.; Danishefsk, S. J. J. Am. Chem. Soc. 1990, 112, 8895.

(d) Wang, J.; Deng, C.; Zhang, Q.; Chai, Y. Org. Lett. 2019, 21, 1103 .

[16] (a) Lou, Y.; Cao, P.; Jia, T.; Zhang, Y.; Wang, M.; Liao, J. Angew. Chem., Int. Ed. 2015, 54, 12134. (b) Reddy, V.; Vijaya, A. R. Org. Lett. 2015, 17, 3390.

(c) Chu, W. D.; Zhang, L. F.; Bao, X.; Zhao, X. H.; Zeng, C.; Du, J. Y.; Zhang, G. B.; Wang, F. X.; Ma, X. Y.; Fan, C. A. Angew. Chem. Int. Ed. 2013, 52, 9229.

(d) Onishi, Y.; Nishimoto, Y.; Yasuda, M.; Baba, A. Org. Lett. 2014 $16,1176$.

(Li, L.; Fan, Y.) 